\title{
Catalytically Asymmetric Pd/Norbornene Catalysis: Enantioselective Synthesis of (+)-Rhazinal, (+)-Rhazinilam and (+)-Kopsiyunnanine C1-3
}

Kun Zhao, Shibo Xu, Chongqing Pan, Xianwei Sui and Zhenhua Gu* University of Science and Technology of China, 96 Jinzhai Road, Hefei, Anhui, China, 230026

zhgu@ustc.edu.cn

\section{Supporting Information}

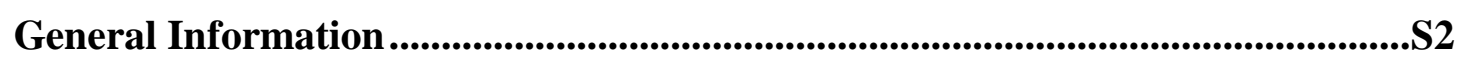

General Procedures for Preparation of Phosphoramidites ..................................S2

Procedures for synthesis of (+)-Rhazinal, (+)-Rhazinilam and (+)-Kopsiyunnanine C1-3 and the analytic data ............................................................S4

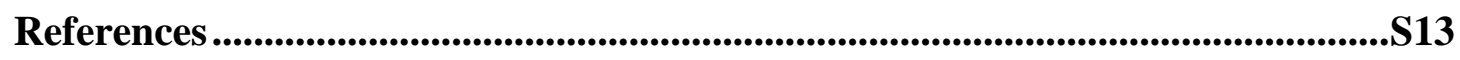

Copies of ${ }^{1} \mathrm{H}$ NMR, ${ }^{13} \mathrm{C}$ NMR and ${ }^{31} \mathrm{P}$ NMR spectra and HPLC traces .............S14 


\section{General Information}

All reactions were carried out under a nitrogen atmosphere in oven or flame-dried glassware, unless the reaction procedure states otherwise. Tetrahydrofuran (THF) was distilled from sodium-benzophenone in a continuous still under an atmosphere of $\mathrm{N}_{2}$. $\mathrm{Et}_{3} \mathrm{~N}$, dichloromethane and toluene were distilled from calcium hydride in a still under an atmosphere of nitrogen. Flash column chromatography was performed using 200-300 mesh silica gel as the stationary phase. ${ }^{1} \mathrm{H}$ and ${ }^{13} \mathrm{C}$ NMR spectra were recorded on a Bruker AC-400 FT spectrometer using solvent residue as an internal reference (7.26 and $77.00 \mathrm{ppm}$ for $\mathrm{CDCl}_{3}, 2.50$ and $39.00 \mathrm{ppm}$ for DMSO- $d_{6}$, respectively). High resolution mass spectra (HRMS) were recorded on a high-resolution mass spectrometer (Waters XEVO-G2 Q-TOF).

\section{Typical Procedures for Preparation of Phosphoramidites}

\section{Synthesis of Compound $\mathrm{L12}^{[1]}$ (Typical procedure A)}

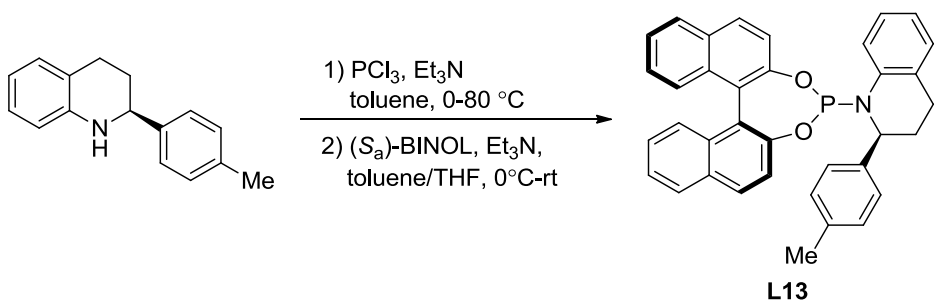

A flame-dried flask was charged with dry toluene $(1.0 \mathrm{~mL})$ and $\mathrm{PCl}_{3}(18.0 \mu \mathrm{L}$, 0.200 mmol, 1.2 equiv), and then cooled to $0{ }^{\circ} \mathrm{C}$. A mixture of (S)-2-(4'-methylphenyl)-1,2,3,4-tetrahydroquinoline $(38.0 \mathrm{mg}, \quad 0.170 \mathrm{mmol}, 1.0$ equiv), toluene $(0.2 \mathrm{~mL})$, and $\mathrm{Et}_{3} \mathrm{~N}(47.0 \mu \mathrm{L}, 0.340 \mathrm{mmol}, 2.0$ equiv) was added dropwise to above mentioned $\mathrm{PCl}_{3}$ solution at $0{ }^{\circ} \mathrm{C}$, and the mixture was heated at 80 ${ }^{\circ} \mathrm{C}$ for $6 \mathrm{~h}$. After being chilled to $-78{ }^{\circ} \mathrm{C}$, a solution of $\left(S_{\mathrm{a}}\right)$-BINOL $(49.0 \mathrm{mg}, 0.170$ mmol, 1.0 equiv) and $\mathrm{Et}_{3} \mathrm{~N}(85.0 \mu \mathrm{L}, 0.610 \mathrm{mmol}, 3.6$ equiv) in dry toluene $(0.6 \mathrm{~mL})$ and THF $(0.2 \mathrm{~mL})$ was added dropwise within $5 \mathrm{~min}$. The resulting solution was stirred at room temperature overnight, then filtered through celite with $\mathrm{Et}_{2} \mathrm{O}$ as eluent. The filtrate was concentrated in vacuo, and the residue was purified by column chromatography on silica gel (hexanes/ethyl acetate/Et ${ }_{3} \mathrm{~N}=100: 1: 0.1$ ) to afford $\mathbf{L 1 3}$ as a white solid $(55.0 \mathrm{mg}, 50 \%)$. $[\alpha]_{D}^{20}+32.3\left(\mathrm{c} 0.69, \mathrm{CHCl}_{3}\right) .{ }^{1} \mathbf{H}$ NMR $(400 \mathrm{MHz}$, $\left.\mathrm{CDCl}_{3}\right) \delta 7.98(\mathrm{~d}, J=8.8 \mathrm{~Hz}, 1 \mathrm{H}), 7.93(\mathrm{~d}, J=8.0 \mathrm{~Hz}, 1 \mathrm{H}), 7.75(\mathrm{~d}, J=8.0 \mathrm{~Hz}, 1 \mathrm{H})$, $7.59(\mathrm{~d}, J=8.4 \mathrm{~Hz}, 1 \mathrm{H}), 7.50(\mathrm{~d}, J=8.8 \mathrm{~Hz}, 1 \mathrm{H}), 7.47-7.35(\mathrm{~m}, 3 \mathrm{H}), 7.32-7.21(\mathrm{~m}$, $4 \mathrm{H}), 7.11(\mathrm{t}, J=7.1 \mathrm{~Hz}, 1 \mathrm{H}), 7.03-6.87(\mathrm{~m}, 4 \mathrm{H}), 6.81(\mathrm{~d}, J=8.0 \mathrm{~Hz}, 2 \mathrm{H}), 6.65(\mathrm{~d}, J$ $=8.8 \mathrm{~Hz}, 1 \mathrm{H}), 4.91(\mathrm{~s}, 1 \mathrm{H}), 2.62(\mathrm{dd}, J=16.8,4.4 \mathrm{~Hz}, 1 \mathrm{H}), 2.46-2.25(\mathrm{~m}, 4 \mathrm{H})$, 2.21-2.09 (m, $1 \mathrm{H}), 2.04-1.88(\mathrm{~m}, 1 \mathrm{H}) .{ }^{13} \mathbf{C ~ N M R}\left(100 \mathrm{MHz}, \mathrm{CDCl}_{3}\right) \delta 149.4(\mathrm{~d}, J=$ $2.0 \mathrm{~Hz}), 148.8,141.1,140.9$, 138.8, 135.6, 132.8, 132.2, 131.5, 130.6, 130.4, 129.8, $129.1,128.6,128.3,128.2,127.1,126.9,126.5,126.1,125.7,124.9,124.4,124.3$, $124.2,122.0,121.8,121.3(\mathrm{~d}, J=2.0 \mathrm{~Hz}), 120.1,119.8,52.5,28.4,22.7,21.1 .{ }^{31} \mathbf{P}$ NMR $\left(162 \mathrm{MHz}, \mathrm{CDCl}_{3}\right) \delta$ 142.1. HRMS (ESI) calcd for $\mathrm{C}_{36} \mathrm{H}_{29} \mathrm{NO}_{2} \mathrm{P}[\mathrm{M}+\mathrm{H}]^{+}$ 


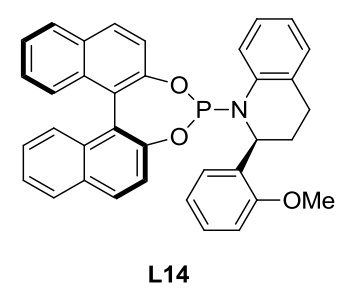

L14 was prepared following the Typical Procedure A

The reaction of $\left(S_{\mathrm{a}}\right)$-BINOL $\left(220.0 \mathrm{mg}, 0.770 \mathrm{mmol}, 1.0\right.$ equiv), $\mathrm{PCl}_{3}(84.0 \mu \mathrm{L}$, 0.930 mmol, 1.2 equiv), (S)-2-(2'-methoxyphenyl)-1,2,3,4-tetrahydroquinoline (185.4 $\mathrm{mg}, 0.770 \mathrm{mmol}, 1.0$ equiv) gave $\mathbf{L 1 4}(171.5 \mathrm{mg}, 40 \%) .[\alpha]_{D}^{20}+116.1$ (c 0.21 , $\left.\mathrm{CHCl}_{3}\right) .{ }^{1} \mathbf{H}$ NMR $\left(400 \mathrm{MHz}, \mathrm{CDCl}_{3}\right) \delta 7.98(\mathrm{~d}, J=8.8 \mathrm{~Hz}, 1 \mathrm{H}), 7.92(\mathrm{~d}, J=8.4 \mathrm{~Hz}$, $1 \mathrm{H}), 7.73(\mathrm{~d}, J=8.0 \mathrm{~Hz}, 1 \mathrm{H}), 7.63(\mathrm{~d}, J=8.0 \mathrm{~Hz}, 1 \mathrm{H}), 7.51(\mathrm{~d}, J=8.4 \mathrm{~Hz}, 1 \mathrm{H})$, 7.46-7.32 (m, $3 \mathrm{H}), 7.24-7.16(\mathrm{~m}, 5 \mathrm{H}), 7.09(\mathrm{~d}, J=8.4 \mathrm{~Hz}, 2 \mathrm{H}), 7.03(\mathrm{~d}, J=6.8 \mathrm{~Hz}$, $1 \mathrm{H}), 6.98(\mathrm{dd}, J=14.4,6.8 \mathrm{~Hz}, 1 \mathrm{H}), 6.78(\mathrm{t}, J=7.2 \mathrm{~Hz}, 1 \mathrm{H}), 6.55(\mathrm{~d}, J=7.6 \mathrm{~Hz}, 1$ H), $6.43(\mathrm{~d}, J=8.8 \mathrm{~Hz}, 1 \mathrm{H}), 5.26(\mathrm{~s}, 1 \mathrm{H}), 2.92(\mathrm{~s}, 3 \mathrm{H}), 2.61(\mathrm{~d}, J=16.4 \mathrm{~Hz}, 1 \mathrm{H})$, 2.44-2.25 (m, $1 \mathrm{H}), 2.05-1.88(\mathrm{~m}, 2 \mathrm{H}) .{ }^{13} \mathbf{C}$ NMR $\left(100 \mathrm{MHz}, \mathrm{CDCl}_{3}\right) \delta 155.7,149.0$ $(\mathrm{d}, J=6.5 \mathrm{~Hz}), 142.1,141.8,132.8,132.3,131.5,130.7,130.4(\mathrm{~d}, J=5.7 \mathrm{~Hz}), 129.9$, $128.5,128.3,127.9,127.7,127.4,127.1,127.0,126.8,126.7,126.5(\mathrm{~d}, J=2.0 \mathrm{~Hz})$, 126.0, 125.5, 124.8, 124.3, 124.2, 124.1, 122.0, 121.6 (d, $J=2.4 \mathrm{~Hz}), 119.64,119.1$, 118.8, 109.5, 53.9, $47.7(\mathrm{~d}, J=2.7 \mathrm{~Hz}), 26.0,23.2 .{ }^{31} \mathbf{P}$ NMR $\left(162 \mathrm{MHz}, \mathrm{CDCl}_{3}\right) \delta$ 142.2. HRMS (ESI) calcd for $\mathrm{C}_{36} \mathrm{H}_{29} \mathrm{NO}_{3} \mathrm{P}[\mathrm{M}+\mathrm{H}]^{+}$554.1885, found 554.1883.

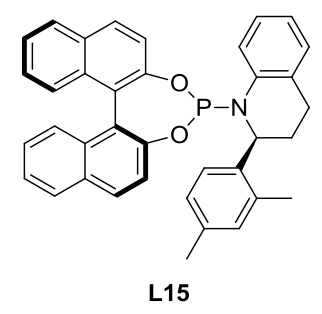

L15 was prepared following the Typical Procedure A

The reaction of $\left(S_{\mathrm{a}}\right)$-BINOL $\left(72.0 \mathrm{mg}, 0.253 \mathrm{mmol}, 1.0\right.$ equiv), $\mathrm{PCl}_{3}(27.0 \mu \mathrm{L}$, 0.300 mmol, 1.2 equiv), (S)-2-(2',4'-dimethylphenyl)-1,2,3,4-tetrahydroquinoline (60.0 mg, $0.770 \mathrm{mmol}, 1.0$ equiv) gave $\mathbf{L 1 5}(78.1 \mathrm{mg}, 56 \%)$. $[\alpha]_{D}^{20}+73.6$ (c 0.44 , $\left.\mathrm{CHCl}_{3}\right) .{ }^{1} \mathbf{H}$ NMR $\left(400 \mathrm{MHz}, \mathrm{CDCl}_{3}\right) \delta 7.99(\mathrm{~d}, J=8.0 \mathrm{~Hz}, 1 \mathrm{H}), 7.93(\mathrm{~d}, J=8.0 \mathrm{~Hz}$, $1 \mathrm{H}), 7.76(\mathrm{~d}, J=8.0 \mathrm{~Hz}, 1 \mathrm{H}), 7.63(\mathrm{~d}, J=8.0 \mathrm{~Hz}, 1 \mathrm{H}), 7.53(\mathrm{~d}, J=8.0 \mathrm{~Hz}, 1 \mathrm{H})$, 7.44-7.37 (m, $3 \mathrm{H}), 7.28-7.19(\mathrm{~m}, 4 \mathrm{H}), 7.12(\mathrm{~d}, J=8.0 \mathrm{~Hz}, 1 \mathrm{H}), 7.07$ (d, $J=8.0 \mathrm{~Hz}$, $1 \mathrm{H}), 6.99(\mathrm{~m}, 2 \mathrm{H}), 6.83(\mathrm{~d}, J=8.0 \mathrm{~Hz}, 1 \mathrm{H}), 6.64(\mathrm{~s}, 1 \mathrm{H}), 6.42(\mathrm{~d}, J=8.0 \mathrm{~Hz}, 1 \mathrm{H})$, $5.21(\mathrm{~s}, 1 \mathrm{H}), 2.64(\mathrm{~d}, J=16.0 \mathrm{~Hz}, 1 \mathrm{H}), 2.43(\mathrm{~m}, 1 \mathrm{H}), 2.35(\mathrm{~s}, 3 \mathrm{H}), 2.11-2.01(\mathrm{~m}, 1$ $\mathrm{H}), 1.79-1.70$ (m, $1 \mathrm{H}), 1.14$ (s, $3 \mathrm{H}) .{ }^{13} \mathbf{C}$ NMR $\left(100 \mathrm{MHz}, \mathrm{CDCl}_{3}\right) \delta 148.9(\mathrm{~d}, J=6.0$ $\mathrm{Hz}), 148.8$, 142.2, 142.0, 137.9, 135.7, 134.2, 132.8, 132.2, 131.5, 131.3, 130.6, 130.4, $129.6,129.0,128.3,128.1,127.1(\mathrm{~d}, J=5.0 \mathrm{~Hz}), 127.0,126.7,126.7,126.1(\mathrm{~d}, J=$ $5.0 \mathrm{~Hz}), 125.7,124.9,124.4,124.2(\mathrm{~d}, J=5.0 \mathrm{~Hz}), 121.9,121.7,121.6,121.4,119.2$, $118.9,49.4,26.8,23.1,21.0,18.1 .{ }^{31} \mathbf{P}$ NMR $\left(162 \mathrm{MHz}, \mathrm{CDCl}_{3}\right) \delta 141.22$. HRMS 
(ESI) calcd for $\mathrm{C}_{37} \mathrm{H}_{31} \mathrm{NO}_{2} \mathrm{P}[\mathrm{M}+\mathrm{H}]^{+}$552.2092, found 552.2095.

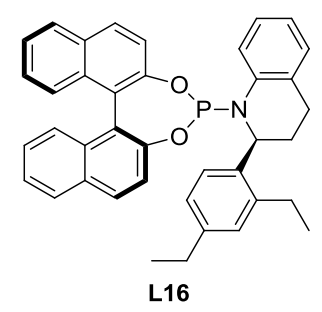

L16 was prepared following the Typical Procedure A

The reaction of $\left(S_{\mathrm{a}}\right)$-BINOL $\left(75.0 \mathrm{mg}, 0.264 \mathrm{mmol}, 1.1\right.$ equiv), $\mathrm{PCl}_{3}(26.0 \mu \mathrm{L}$, 0.290 mmol, 1.2 equiv), (S)-2-(2',4'-diethylphenyl)-1,2,3,4-tetrahydroquinoline (63.0 mg, 0.240 mmol, 1.0 equiv) gave $\mathbf{L 1 6}(68.8 \mathrm{mg}, 50 \%)$. [ $\alpha]_{D}^{20}+32.5$ (c $\left.0.48, \mathrm{CHCl}_{3}\right)$. ${ }^{1} \mathbf{H}$ NMR $\left(400 \mathrm{MHz}, \mathrm{CDCl}_{3}\right) \delta 7.99(\mathrm{~d}, J=8.8 \mathrm{~Hz}, 1 \mathrm{H}), 7.93(\mathrm{~d}, J=8.0 \mathrm{~Hz}, 1 \mathrm{H})$, $7.72(\mathrm{~d}, J=8.0 \mathrm{~Hz}, 1 \mathrm{H}), 7.62(\mathrm{~d}, J=8.0 \mathrm{~Hz}, 1 \mathrm{H}), 7.54(\mathrm{~d}, J=8.8 \mathrm{~Hz}, 1 \mathrm{H})$, 7.44-7.34 (m, $3 \mathrm{H}), 7.28-7.16(\mathrm{~m}, 3 \mathrm{H}), 7.09$ (d, $J=8.8 \mathrm{~Hz}, 2 \mathrm{H}), 7.03$ (t, $J=8.4 \mathrm{~Hz}, 2$ $\mathrm{H}), 6.87(\mathrm{~d}, J=8.0 \mathrm{~Hz}, 1 \mathrm{H}), 6.69(\mathrm{~s}, 1 \mathrm{H}), 6.42(\mathrm{~d}, J=8.8 \mathrm{~Hz}, 1 \mathrm{H}), 5.38(\mathrm{~s}, 1 \mathrm{H})$, $2.67(\mathrm{~m}, 3 \mathrm{H}), 2.54-2.43(\mathrm{~m}, 1 \mathrm{H}), 2.13(\mathrm{~m}, 1 \mathrm{H}), 1.86(\mathrm{dd}, J=15.2,7.6 \mathrm{~Hz}, 1 \mathrm{H})$, $1.82-1.71(\mathrm{~m}, 1 \mathrm{H}), 1.34(\mathrm{t}, J=7.6 \mathrm{~Hz}, 3 \mathrm{H}), 1.24(\mathrm{~m}, 2 \mathrm{H}), 0.57(\mathrm{t}, J=7.6 \mathrm{~Hz}, 3 \mathrm{H})$. ${ }^{13} \mathrm{C}$ NMR $\left(100 \mathrm{MHz}, \mathrm{CDCl}_{3}\right) \delta 148.9(\mathrm{~d}, J=6.6 \mathrm{~Hz}), 148.8,142.3,142.2,142.1$, 139.6, 137.7, 132.8, 132.2, 131.5, 130.4, 130.3, 129.4, 128.8, 128.3, 128.1, 127.6, $127.1(\mathrm{~d}, J=3.8 \mathrm{~Hz}), 126.8(\mathrm{~d}, J=4.3 \mathrm{~Hz}), 126.7,126.1,125.6,124.9,124.6,124.3$, $124.3(\mathrm{~d}, J=5.2 \mathrm{~Hz}), 121.9$ (d, $J=8.5 \mathrm{~Hz}), 121.6,121.3,119.3,119.0,53.4,49.0$, 28.6, 28.1, 23.4, 23.4 15.9, 13.5. ${ }^{31} \mathbf{P}$ NMR $\left(162 \mathrm{MHz}, \mathrm{CDCl}_{3}\right) \delta 140.6$. HRMS (ESI) calcd for $\mathrm{C}_{39} \mathrm{H}_{35} \mathrm{NO}_{2} \mathrm{P}[\mathrm{M}+\mathrm{H}]^{+}$580.2405, found 580.2404.

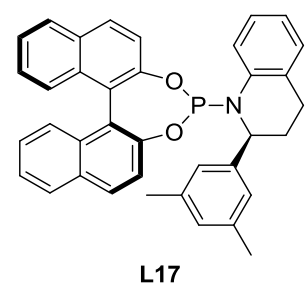

L17 was prepared following the Typical Procedure A

The reaction of $\left(S_{\mathrm{a}}\right)$-BINOL (72.0 mg, $0.253 \mathrm{mmol}, 1.0$ equiv), $\mathrm{PCl}_{3}(27.0 \mu \mathrm{L}, 0.300$ mmol, 1.2 equiv), (S)-2-(3',5'-dimethylphenyl)-1,2,3,4-tetrahydroquinoline (60.0 mg, 0.770 mmol, 1.0 equiv) gave $\mathbf{L 1 7}(44.6 \mathrm{mg}, 32 \%) .[\alpha]_{D}^{20}+52.6\left(\mathrm{c} 0.24, \mathrm{CHCl}_{3}\right) .{ }^{1} \mathbf{H}$ NMR $\left(400 \mathrm{MHz}, \mathrm{CDCl}_{3}\right) \delta 7.97(\mathrm{~d}, J=8.0 \mathrm{~Hz}, 1 \mathrm{H}), 7.92(\mathrm{~d}, J=8.0 \mathrm{~Hz}, 1 \mathrm{H}), 7.74$ $(\mathrm{d}, J=8.0 \mathrm{~Hz}, 1 \mathrm{H}), 7.58(\mathrm{~d}, J=8.0 \mathrm{~Hz}, 1 \mathrm{H}), 7.49(\mathrm{~d}, J=8.0 \mathrm{~Hz}, 1 \mathrm{H}), 7.44-7.36(\mathrm{~m}$, $3 \mathrm{H}), 7.31-7.22(\mathrm{~m}, 3 \mathrm{H}), 7.20(\mathrm{~d}, J=8.0 \mathrm{~Hz}, 1 \mathrm{H}), 7.10(\mathrm{t}, J=8.0 \mathrm{~Hz}, 1 \mathrm{H}), 7.00(\mathrm{~d}, J$ $=8.0 \mathrm{~Hz}, 1 \mathrm{H}), 6.94(\mathrm{~d}, J=4.0 \mathrm{~Hz}, 1 \mathrm{H}), 6.82(\mathrm{~s}, 1 \mathrm{H}), 6.58(\mathrm{~d}, J=12.0 \mathrm{~Hz}, 1 \mathrm{H})$, $6.47(\mathrm{~s}, 2 \mathrm{H}), 4.88(\mathrm{~s}, 1 \mathrm{H}), 2.61(\mathrm{~d}, J=16.0 \mathrm{~Hz}, 1 \mathrm{H}), 2.43(\mathrm{~m}, 1 \mathrm{H}), 2.18(\mathrm{~s}, 6 \mathrm{H})$, $2.12(\mathrm{~m}, 1 \mathrm{H}), 1.92(\mathrm{~m}, 1 \mathrm{H}) .{ }^{13} \mathbf{C}$ NMR $\left(100 \mathrm{MHz}, \mathrm{CDCl}_{3}\right) \delta 149.3,148.8,142.1$, $141.3,141.0,137.2,132.8,132.2,131.5,130.6,130.4,129.7,128.8,128.3,128.1$, $127.8,127.2$, 127.1, 126.9, 126.5, 126.1, 125.7, 124.9, 124.4, 124.4, 124.3, 124.2, 122.0, 121.9, 121.8, 121.3, 119.9, 119.6, 52.6, 28.6, 22.8, 21.4. ${ }^{31} \mathbf{P}$ NMR (162 MHz, 
$\left.\mathrm{CDCl}_{3}\right) \delta$ 142.2. HRMS (ESI) calcd for $\mathrm{C}_{37} \mathrm{H}_{31} \mathrm{NO}_{2} \mathrm{P}[\mathrm{M}+\mathrm{H}]^{+}$552.2092, found 552.2087.

Procedures for synthesis of (+)-Rhazinal, (+)-Rhazinilam and (+)-Kopsiyunnanine C1-3

The synthesis of (+)-Rhazinal ${ }^{[2]}$ and (+)-Kopsiyunnanine $\mathrm{C3}^{[3]}$

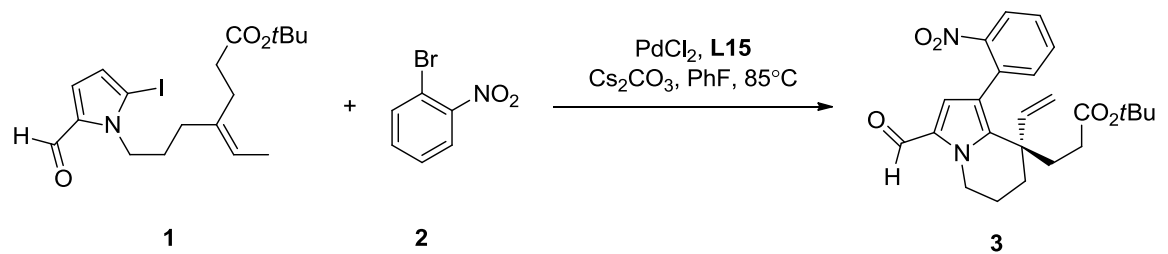

A mixture of 1 (0.300 g, $0.690 \mathrm{mmol}, 1.0$ equiv), $\mathrm{PdCl}_{2}$ (12.2 mg, $0.069 \mathrm{mmol}, 10$ mol\%), L15 (83.8 mg, $0.152 \mathrm{mmol}, 22 \mathrm{~mol} \%), \mathrm{Cs}_{2} \mathrm{CO}_{3}$ (0.562 g, $1.73 \mathrm{mmol}, 2.5$ equiv), norbornene ( $0.389 \mathrm{~g}, 4.14 \mathrm{mmol}, 6.0$ equiv), 2 ( $0.836 \mathrm{~g}, 4.14 \mathrm{mmol}, 6.0$ equiv) in $\mathrm{PhF}(7.0 \mathrm{~mL})$ in a schlenk tube was stirred at $85^{\circ} \mathrm{C}$ for $20 \mathrm{~h}$. After being cooled to room temperature, the mixture was filtered through a plug of celite eluting with ethyl acetate $(20.0 \mathrm{~mL})$. The filtrate was washed with brine $(15.0 \mathrm{~mL})$, dried over sodium sulfate, and concentrated. The crude product was purified by column chromatography (10\% ethyl acetate/hexanes and 50\% dichloromethane/hexanes) to give $\mathbf{3}$ (190.0 mg, $65 \%, 88 \%$ ee.). $[\alpha]_{D}^{20}-91.2$ (c $0.37, \mathrm{CHCl}_{3}$ ). HPLC conditions: Chiralcel AD-H, isopropanol/hexane $=15: 85$, flow: $1.0 \mathrm{~mL} / \mathrm{min}, \lambda=294 \mathrm{~nm} .{ }^{1} \mathbf{H}$ NMR $(400 \mathrm{MHz}$, CDCl3) $\delta 9.46(\mathrm{~s}, 1 \mathrm{H}), 7.82(\mathrm{~d}, J=8.0 \mathrm{~Hz}, 1 \mathrm{H}), 7.64-7.37(\mathrm{~m}, 3 \mathrm{H}), 6.79(\mathrm{~s}, 1 \mathrm{H})$, $5.80(\mathrm{q}, J=9.2 \mathrm{~Hz}, 1 \mathrm{H}), 5.15(\mathrm{bs}, 1 \mathrm{H}), 4.76(\mathrm{bs}, 1 \mathrm{H}), 4.72(\mathrm{~d}, J=17.2 \mathrm{~Hz}, 1 \mathrm{H})$, 4.19-4.00 (m, $1 \mathrm{H}), 2.21-1.99(\mathrm{~m}, 3 \mathrm{H}), 1.99-1.83(\mathrm{~m}, 2 \mathrm{H}), 1.83-1.73(\mathrm{~m}, 1 \mathrm{H})$, 1.73-1.60 (m, $2 \mathrm{H}), 1.33$ (s, $9 \mathrm{H})$.

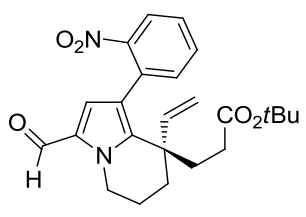

3

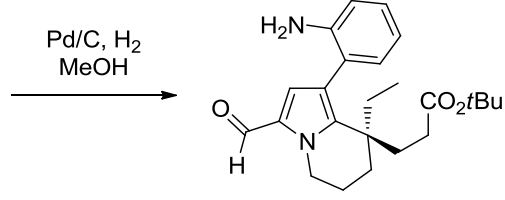

4

A mixture of $\mathrm{Pd} / \mathrm{C}(12.0 \mathrm{mg}, 10 \%$ palladium on carbon) and 3 (40.0 mg, 0.094 mmol) in methanol $(3.0 \mathrm{~mL})$ in a schlenk tube was purged with $\mathrm{H}_{2}$ through a balloon, and stirred under $1 \mathrm{~atm}$ of $\mathrm{H}_{2}$ at room temperature for $4 \mathrm{~h}$. The mixture was filtered through a plug of celite eluting ethyl acetate $(10.0 \mathrm{~mL})$, and the filtrate was concentrated. The crude product was purified by column chromatography ( $80 \%$ ethyl acetate/hexanes) to give $4(35.0 \mathrm{mg}, 94 \%) .[\alpha]_{D}^{20}-72.2\left(\mathrm{c} 0.32, \mathrm{CHCl}_{3}\right) .{ }^{\mathbf{1}} \mathbf{H} \mathbf{~ N M R}$ (400 MHz, CDCl3) $\delta 9.42(\mathrm{~s}, 1 \mathrm{H}), 7.21-7.12(\mathrm{~m}, 1 \mathrm{H}), 7.07$ (bd, $J=7.2 \mathrm{~Hz}, 1 \mathrm{H})$, 6.87-6.68 (m, $3 \mathrm{H}), 4.61-4.41(\mathrm{~m}, 1 \mathrm{H}), 4.41-4.18(\mathrm{~m}, 1 \mathrm{H}), 2.27-2.11(\mathrm{~m}, 1 \mathrm{H})$, 2.11-1.99 (m, $1 \mathrm{H}), 1.99-1.83$ (m, $2 \mathrm{H}), 1.83-1.68$ (m, $2 \mathrm{H}), 1.68-1.54$ (m, $2 \mathrm{H}), 1.41$ (d, $J=24.4 \mathrm{~Hz}, 11 \mathrm{H}), 0.86-0.62(\mathrm{~m}, 3 \mathrm{H})$ 


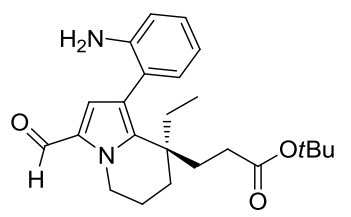

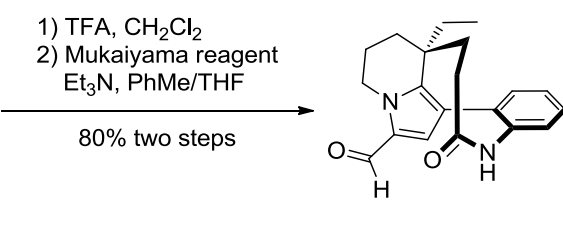

(+)-rhazinal (5)

Trifluoroacetic acid $(1.0 \mathrm{~mL})$ was added to the solution of the ester $4(41.4 \mathrm{mg}$, $0.122 \mathrm{mmol})$ in dichloromethane $(2.0 \mathrm{~mL})$ dropwise. After being stirred at room temperature for $4 \mathrm{~h}$, the mixture was concentrated in vacuo. The obtained amino acid was used in next step without further purification.

To a solution of 2-chloro-1-methylpyridinium iodide $(0.360 \mathrm{~g}, 1.22 \mathrm{mmol}, 10.0$ equiv) and $\mathrm{Et}_{3} \mathrm{~N}(0.60 \mathrm{~mL}, 3.66 \mathrm{mmol}, 30.0$ equiv) in toluene $(20.0 \mathrm{~mL})$ was added a solution of the above amino acid in $\mathrm{THF} /$ toluene $(3 / 2,20.0 \mathrm{~mL})$ via a syringe pump over $15 \mathrm{~h}$. The solid was filtered off after stirring for another $2 \mathrm{~h}$ and the filtrate was concentrated and purified by column chromatography (70\% ethyl acetate/ hexanes) to give (+)-rhazinal (5) $(31.6 \mathrm{mg}, 80 \% \text {, two steps). [ } \alpha]_{D}^{20}+154.3\left(\mathrm{c} 0.2, \mathrm{CHCl}_{3}\right)$ ref. $^{[4]}$ $\left.[\alpha]_{D}^{20}-187\left(\mathrm{c} 0.2, \mathrm{CHCl}_{3}\right)\right\} .{ }^{1} \mathbf{H}$ NMR $\left(400 \mathrm{MHz}, \mathrm{CDCl}_{3}\right) \delta 9.39(\mathrm{~s}, 1 \mathrm{H}), 7.53-7.31$ (m, 3 H), 7.30-7.21(br, 1 H), 6.79 (s, 1 H), 6.55 (s, 1 H), 4.78 (dd, $J=14.0,5.2 \mathrm{~Hz}, 1$ H), $3.98(\mathrm{td}, J=13.2,5.1 \mathrm{~Hz}, 1 \mathrm{H}), 2.57-2.35(\mathrm{~m}, 2 \mathrm{H}), 2.27-2.11(\mathrm{~m}, 1 \mathrm{H}), 2.08-1.89$ $(\mathrm{m}, 2 \mathrm{H}), 1.76(\mathrm{td}, J=12.0,4.0 \mathrm{~Hz}, 1 \mathrm{H}), 1.69(\mathrm{~s}, 1 \mathrm{H}), 1.60-1.44(\mathrm{~m}, 2 \mathrm{H}), 1.35-1.16$ $(\mathrm{m}, 1 \mathrm{H}), 0.71(\mathrm{t}, J=7.2 \mathrm{~Hz}, 3 \mathrm{H}) ;{ }^{13} \mathbf{C} \mathbf{N M R}\left(100 \mathrm{MHz}, \mathrm{CDCl}_{3}\right) \delta 178.8,176.6,141.3$, 138.2, 137.5, 131.3, 130.2, 129.0,127.7, 127.3, 125.5, 120.4, 46.3, 39.6, 36.4, 31.9, 29.8, 28.0, 18.5, 8.2; HRMS (ACPI) calcd for $\mathrm{C}_{20} \mathrm{H}_{23} \mathrm{O}_{2} \mathrm{~N}_{2}[\mathrm{M}+\mathrm{H}]^{+} 323.1754$, found 323.1762 .

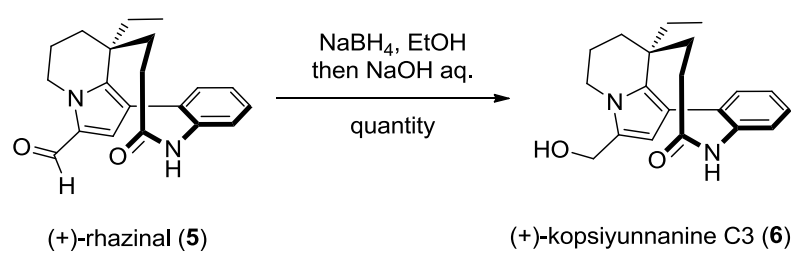

To a solution of $5(13.0 \mathrm{mg} 0.040 \mathrm{mmol})$ in dry $\mathrm{EtOH}(1.50 \mathrm{ml})$ was added $\mathrm{NaBH}_{4}$ (26.0 $\mathrm{mg}, 0.690 \mathrm{mmol}$ ) and was stirred for $1 \mathrm{~h}$ at room temperature. The reaction was quenched with aqueous $\mathrm{NaOH}$ solution and extracted with ethyl acetate. The organic layers were combined, dried over sodium sulfate, and concentrated. The crude product was purified by column chromatography $(15 \% \mathrm{MeOH} / \mathrm{DCM})$ to give (+)-kopsiyunnanine C3 (13.0 mg, quant). $[\alpha]_{D}^{20}+360.2\left(\mathrm{c} 0.05, \mathrm{CHCl}_{3}\right)\left(\right.$ ref. $^{[3]}[\alpha]_{D}^{19}$ -433 (c 0.05, $\left.\left.\mathrm{CHCl}_{3}\right)\right) .{ }^{1} \mathbf{H}$ NMR $\left(400 \mathrm{MHz}, \mathrm{DMSO}_{-} \mathrm{d}_{6}\right) \delta 8.72(\mathrm{~s}, 1 \mathrm{H}), 7.35-7.30(\mathrm{~m}$, $1 \mathrm{H}), 7.27-7.25(\mathrm{~m}, 2 \mathrm{H}), 7.10(\mathrm{~d}, J=7.6 \mathrm{~Hz}, 1 \mathrm{H}), 5.54(\mathrm{~s}, 1 \mathrm{H}), 4.83(\mathrm{t}, J=5.2 \mathrm{~Hz}, 1$ H), $4.33(\mathrm{~d}, J=5.2 \mathrm{~Hz}, 2 \mathrm{H}), 4.07$ (dd, $J=12.0,3.6 \mathrm{~Hz}, 1 \mathrm{H}), 3.63$ (td, $J=12.4,4.8$ $\mathrm{Hz}, 1 \mathrm{H}), 2.26-2.15$ (m, $2 \mathrm{H}), 1.99-1.96(\mathrm{~m}, 1 \mathrm{H}), 1.87$ (d, $J=9.6 \mathrm{~Hz}, 1 \mathrm{H}), 1.84-1.73$ $(\mathrm{m}, 1 \mathrm{H}), 1.60(\mathrm{dd}, J=12.8,3.0 \mathrm{~Hz}, 1 \mathrm{H}), 1.50-1.38(\mathrm{~m}, 2 \mathrm{H}), 1.33(\mathrm{dd}, J=14.0,6.8$ $\mathrm{Hz}, 1 \mathrm{H}), 1.26-1.20(\mathrm{~m}, 1 \mathrm{H}), 0.62(\mathrm{t}, J=7.2 \mathrm{~Hz}, 3 \mathrm{H}) .{ }^{13} \mathbf{C}$ NMR $(100 \mathrm{MHz}$, DMSO-d $\left.{ }_{6}\right) \delta 174.9,139.5,138.9,131.0,130.6,129.5,127.8,126.5,126.2,116.0$, 
109.9, 55.0, 43.0, 38.4, 35.8, 32.5, 29.9, 27.9, 18.8, 8.0. HRMS (ESI) calcd for $\mathrm{C}_{20} \mathrm{H}_{25} \mathrm{O}_{2} \mathrm{~N}_{2}[\mathrm{M}+\mathrm{H}]^{+}$325.1916, found 325.1917.

\section{The synthesis of (+)-Rhazinilam}

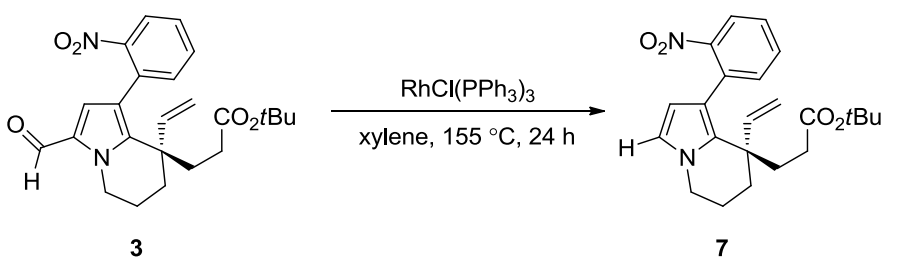

To a solution of $\mathbf{3}(80.0 \mathrm{mg}, 0.189 \mathrm{mmol}, 1.0$ equiv) in xylene was added $\mathrm{RhCl}\left(\mathrm{PPh}_{3}\right)_{3}(175.0 \mathrm{mg}, 0.189 \mathrm{mmol}, 1.0$ equiv) in a tube capped with a screw cap. The mixture was stirred at $155{ }^{\circ} \mathrm{C}$ for $24 \mathrm{~h}$. After cooling to room temperature the solid was filtered off and the filtrate was concentrated and purified by column chromatography (5\% ethyl acetate/hexanes) to give $7(59.7 \mathrm{mg}, 80 \%)$. $[\alpha]_{D}^{20}-88.7$ (c $\left.0.24, \mathrm{CHCl}_{3}\right) .{ }^{1} \mathbf{H}$ NMR $\left(400 \mathrm{MHz}, \mathrm{CDCl}_{3}\right) \delta 7.68(\mathrm{~d}, J=8.0 \mathrm{~Hz}, 1 \mathrm{H}), 7.59(\mathrm{~d}, J=$ $7.6 \mathrm{~Hz}, 1 \mathrm{H}), 7.42$ (t, $J=7.6 \mathrm{~Hz}, 1 \mathrm{H}), 7.34(\mathrm{t}, J=7.6 \mathrm{~Hz}, 1 \mathrm{H}), 6.57(\mathrm{~s}, 1 \mathrm{H}), 6.01(\mathrm{~s}$, $1 \mathrm{H}), 5.84(\mathrm{dd}, J=17.2,6.4 \mathrm{~Hz}, 1 \mathrm{H}), 5.12(\mathrm{~d}, J=10.4 \mathrm{~Hz}, 1 \mathrm{H}), 4.71(\mathrm{~d}, J=17.6 \mathrm{~Hz}$, $1 \mathrm{H}), 4.01(\mathrm{bd}, J=5.2 \mathrm{~Hz}, 1 \mathrm{H}), 3.87(\mathrm{dt}, J=11.6,4.8 \mathrm{~Hz}, 1 \mathrm{H}), 2.28-2.08(\mathrm{~m}, 3 \mathrm{H})$, $1.99-1.92(\mathrm{~m}, 1 \mathrm{H}), 1.84(\mathrm{~d}, J=12.6 \mathrm{~Hz}, 1 \mathrm{H}), 1.76(\mathrm{t}, J=13.2 \mathrm{~Hz}, 1 \mathrm{H}), 1.66-1.56(\mathrm{~m}$, $2 \mathrm{H}), 1.31$ (s, $9 \mathrm{H}) .{ }^{13} \mathbf{C}$ NMR $\left(100 \mathrm{MHz}, \mathrm{CDCl}_{3}\right) \delta 173.0,150.4,145.9,133.5,132.6$, 130.9 , 128.3, 127.1, 123.4, 119.7, 115.1, 114.6, 110.0, 79.8, 77.4, 77.0, 76.7, 46.0, 42.9, 32.3, 31.4, 30.41, 28.0, 19.1. HRMS (ESI) calcd for $\mathrm{C}_{23} \mathrm{H}_{29} \mathrm{O}_{4} \mathrm{~N}_{2}[\mathrm{M}+\mathrm{H}]^{+}$ 397.2127, found 397.2124 .
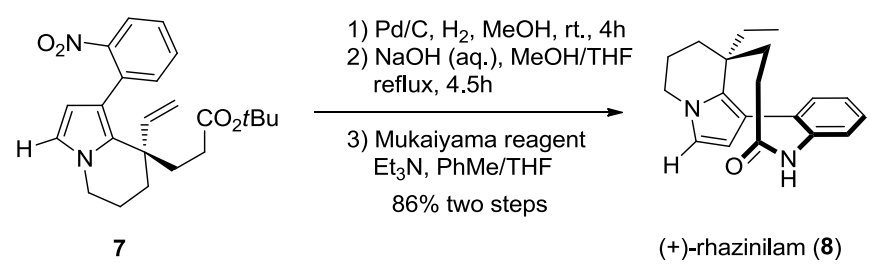

A mixture of $\mathrm{Pd} / \mathrm{C}(18.0 \mathrm{mg}, 10 \%$ palladium on carbon) and $3(48.0 \mathrm{mg}, 0.121$ mmol) in methanol $(4.0 \mathrm{~mL})$ in a schlenk tube was purged with $\mathrm{H}_{2}$ through a balloon, and stirred under $1 \mathrm{~atm}$ of $\mathrm{H}_{2}$ at room temperature for $4 \mathrm{~h}$. The mixture was filtered through a plug of celite eluted with ethyl acetate $(10.0 \mathrm{~mL})$, and the filtrate was evaporated. The crude product was used in next step without further purification.

A mixture of the above ester, $\mathrm{NaOH}(0.102 \mathrm{~g}, 3.03 \mathrm{mmol}), \mathrm{MeOH}(1.0 \mathrm{~mL})$, THF $(1.0 \mathrm{~mL})$, and water $(1.0 \mathrm{~mL})$ was heated at reflux for $4.5 \mathrm{~h}$. After being cooled to room temperature, the mixture was carefully adjusted to $\mathrm{pH}$ 6-7 by $1 \mathrm{~N} \mathrm{HCl}$ and then extracted with ethyl acetate, washed with brine and dried over sodium sulfate. After concentration, $2 \mathrm{~mL}$ of dry toluene was added to the residue quickly, then solvent was removed by evaporation; this was repeated three times. The obtained amino acid was used in next step without further purification.

To a solution of 2-chloro-1-methylpyridinium iodide $(0.360 \mathrm{~g}, 1.22 \mathrm{mmol}, 10.0$ equiv) and $\mathrm{Et}_{3} \mathrm{~N}(0.60 \mathrm{~mL}, 3.66 \mathrm{mmol}, 30.0$ equiv) in toluene $(20.0 \mathrm{~mL})$ was added a solution of the above amino acid in $\mathrm{THF} /$ toluene $(3 / 2,20.0 \mathrm{~mL})$ via a syringe pump 
over $15 \mathrm{~h}$. After stirring for additional $2 \mathrm{~h}$ the solid was filtered off and the filtrate was concentrated and purified by column chromatography (50\% ethyl acetate/hexanes) to give (+)-rhazinilam (8) $(30.6 \mathrm{mg}, 86 \%, 3$ steps $) . \quad[\alpha]_{D}^{20}+348.6\left(\mathrm{c} 0.96, \mathrm{CHCl}_{3}\right)\left\{\right.$ ref. $^{[4]}$ $\left.[\alpha]_{D}^{20}-421\left(\mathrm{c} 1.00, \mathrm{CHCl}_{3}\right)\right\} .{ }^{1} \mathbf{H}$ NMR $\left(400 \mathrm{MHz}, \mathrm{CDCl}_{3}\right) \delta 7.43(\mathrm{dd}, J=7.6,1.6 \mathrm{~Hz}$, $1 \mathrm{H}), 7.36-7.28(\mathrm{~m}, 2 \mathrm{H}), 7.20(\mathrm{dd}, J=7.2,1.2 \mathrm{~Hz}, 1 \mathrm{H}), 6.66(\mathrm{~s}, 1 \mathrm{H}), 6.50(\mathrm{~d}, J=2.4$ $\mathrm{Hz}, 1 \mathrm{H}), 5.75(\mathrm{~d}, J=2.8 \mathrm{~Hz}, 1 \mathrm{H}), 4.01(\mathrm{dd}, J=12.4,5.2 \mathrm{~Hz}, 1 \mathrm{H}), 3.79(\mathrm{td}, J=12.0$, $4.8 \mathrm{~Hz}, 1 \mathrm{H}), 2.41(\mathrm{td}, J=19.2,6.0 \mathrm{~Hz}, 2 \mathrm{H}), 2.30-2.18(\mathrm{~m}, 1 \mathrm{H}), 1.95(\mathrm{dd}, J=13.2$, $8.0 \mathrm{~Hz}, 1 \mathrm{H}), 1.88-1.83(\mathrm{~m}, 1 \mathrm{H}), 1.76-1.68$ (m, $2 \mathrm{H}), 1.56-1.47$ (m, $2 \mathrm{H}), 1.29-1.20$ $(\mathrm{m}, 1 \mathrm{H}), 0.71(\mathrm{t}, J=7.6 \mathrm{~Hz}, 3 \mathrm{H}) \cdot{ }^{13} \mathbf{C}$ NMR $\left(100 \mathrm{MHz}, \mathrm{CDCl}_{3}\right) \delta 177.3,140.4$, 138.0, 131.4, 130.5, 128.0, 127.2, 126.8, 119.1, 117.3, 109.5, 46.0, 38.8, 36.6, 33.0, 30.1, 28.1, 19.4, 8.1. HRMS (ESI) calcd for $\mathrm{C}_{19} \mathrm{H}_{23} \mathrm{ON}_{2}[\mathrm{M}+\mathrm{H}]^{+} 295.1810$, found 295.1813.

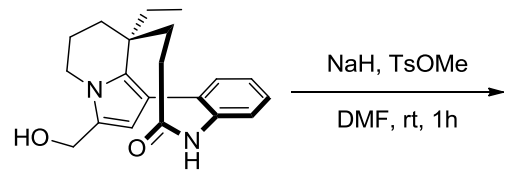

6

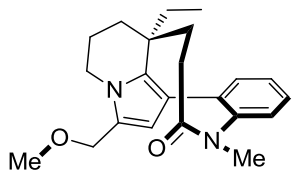

N,O-diMe-6

To a solution of $6(10.0 \mathrm{mg}, 0.031 \mathrm{mmol}, 1.0$ equiv) in DMF (1.0 mL) was added $\mathrm{NaH}$ (3.1 mg, $0.062 \mathrm{mmol}, 2.5$ equiv) and TsOMe (10.0 $\mu \mathrm{L}, 0.062 \mathrm{mmol}, 2.0$ equiv). The mixture was stirred at room temperature for $1 \mathrm{~h}$ and then diluted with ethyl acetate, washed with $\mathrm{H}_{2} \mathrm{O}$, brine and dried over sodium sulfate. The crude product was purified by column chromatography (50\% EA/PE) to give $N, O$-diMe-6 (10.6 mg, 99\%). $[\alpha]_{D}^{20}+311.2\left(\mathrm{c} 0.09, \mathrm{CHCl}_{3}\right) .{ }^{1} \mathbf{H}$ NMR $\left(400 \mathrm{MHz}, \mathrm{CDCl}_{3}\right) \delta 7.40-7.34(\mathrm{~m}, 2$ H), 7.28-7.21 (m, $2 \mathrm{H}), 5.82(\mathrm{~s}, 1 \mathrm{H}), 4.32$ (d, $J=12.4 \mathrm{~Hz}, 1 \mathrm{H}), 4.27$ (d, $J=12.4 \mathrm{~Hz}$, $1 \mathrm{H}), 4.06(\mathrm{dd}, J=12.4,5.2 \mathrm{~Hz}, 1 \mathrm{H}), 3.66(\mathrm{td}, J=12.4,4.8 \mathrm{~Hz}, 1 \mathrm{H}), 3.27(\mathrm{~s}, 3 \mathrm{H})$, 2.93 (s, $3 \mathrm{H}), 2.51-2.37$ (m, $2 \mathrm{H}), 2.25-2.14(\mathrm{~m}, 1 \mathrm{H}), 1.99-1.93(\mathrm{~m}, 1 \mathrm{H}), 1.90-1.85$ $(\mathrm{m}, 1 \mathrm{H}), 1.67(\mathrm{dt}, J=13.6,2.4 \mathrm{~Hz}, 1 \mathrm{H}), 1.49-1.37(\mathrm{~m}, 3 \mathrm{H}), 1.19-1.13(\mathrm{~m}, 1 \mathrm{H}), 0.67$ $(\mathrm{t}, J=7.6 \mathrm{~Hz}, 3 \mathrm{H}) .{ }^{13} \mathrm{C}$ NMR $\left(100 \mathrm{MHz}, \mathrm{CDCl}_{3}\right) \delta 175.5,144.9,138.9,132.2,131.6$, 129.0, 128.6, 126.9, 125.9, 115.6, 110.5, 56.7, 43.4, 39.1, 37.3, 36.5, 32.6, 30.1, 29.3, 19.1, 8.2. HRMS (ESI) calcd for $\mathrm{C}_{22} \mathrm{H}_{29} \mathrm{O}_{2} \mathrm{~N}_{2}[\mathrm{M}+\mathrm{H}]^{+} 353.2229$, found 353.2232.

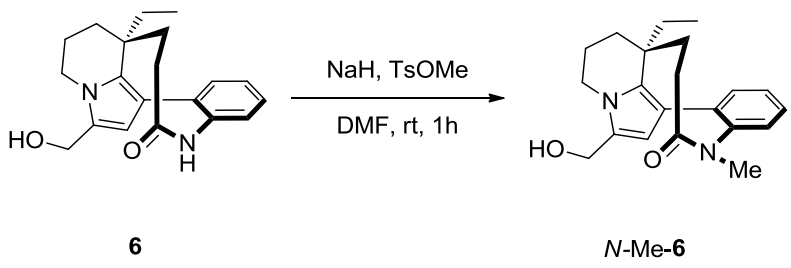

To a solution of 6 (10.0 mg, $0.031 \mathrm{mmol}, 1.0$ equiv) in DMF (1.0 mL) was added $\mathrm{NaH}$ (1.3 mg, $0.031 \mathrm{mmol}, 1.0$ equiv) and TsOMe (5.0 $\mu \mathrm{L}, 0.031 \mathrm{mmol}, 1.0$ equiv). The mixture was stirred at room temperature for $1 \mathrm{~h}$ and then diluted with ethyl acetate, washed with $\mathrm{H}_{2} \mathrm{O}$, brine and dried over sodium sulfate. The crude product was purified by column chromatography (80\% EA/PE) to give $N$-Me-6 (10.0 mg, 99\%). $[\alpha]_{D}^{20}+290.5\left(\mathrm{c} 0.11, \mathrm{CHCl}_{3}\right) .{ }^{1} \mathbf{H}$ NMR $\left(400 \mathrm{MHz}, \mathrm{CDCl}_{3}\right) \delta 7.38(\mathrm{t}, J=7.2$ $\mathrm{Hz}, 2 \mathrm{H}), 7.26(\mathrm{dd}, J=18.8,4.4 \mathrm{~Hz}, 2 \mathrm{H}), 5.79$ (s, $1 \mathrm{H}), 4.48(\mathrm{dd}, J=12.8,6.0 \mathrm{~Hz}, 2$ 
H), $4.20(\mathrm{dd}, J=12.4,5.2 \mathrm{~Hz}, 1 \mathrm{H}), 3.70(\mathrm{td}, J=12.4,4.8 \mathrm{~Hz}, 1 \mathrm{H}), 2.94(\mathrm{~s}, 3 \mathrm{H})$, 2.50-2.37 (m, $2 \mathrm{H}), 2.22-2.12(\mathrm{~m}, 2 \mathrm{H}), 1.97$ (t, $J=7.2 \mathrm{~Hz}, 1 \mathrm{H}), 1.69$ (td, $J=13.6$, $2.8 \mathrm{~Hz}, 1 \mathrm{H}), 1.51-1.48(\mathrm{~m}, 1 \mathrm{H}), 1.44-1.35(\mathrm{~m}, 2 \mathrm{H}), 1.22-1.13(\mathrm{~m}, 1 \mathrm{H}), 0.69(\mathrm{t}, J=$ $7.2 \mathrm{~Hz}, 3 \mathrm{H}) .{ }^{13} \mathrm{C}$ NMR $\left(100 \mathrm{MHz}, \mathrm{CDCl}_{3}\right) \delta 175.4,144.9,138.8,132.1,131.6,129.0$, 128.5, 126.8, 125.8, 115.5, 110.5, 77.0, 56.6, 43.3, 39.1, 37.3, 36.4, 32.5, 30.1, 29.3, 19.1, 8.2. HRMS (ESI) calcd for $\mathrm{C}_{21} \mathrm{H}_{27} \mathrm{O}_{2} \mathrm{~N}_{2}[\mathrm{M}+\mathrm{H}]^{+} 339.2073$, found 339.2075.

\section{The synthesis of (+)-Kopsiyunnanine $\mathrm{C} 1$ and $\mathrm{C2}$}

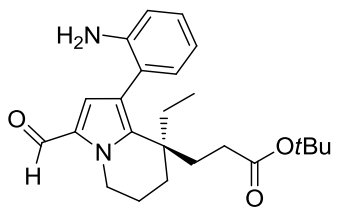

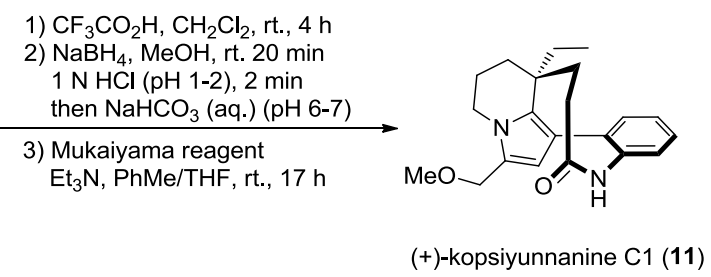

Trifluoroacetic acid $(1.0 \mathrm{~mL})$ was added to the solution of the ester 4 (41.4 $\mathrm{mg}$, $0.122 \mathrm{mmol})$ in dichloromethane $(2.0 \mathrm{~mL})$ dropwise. After being stirred at room temperature for $4 \mathrm{~h}$, the mixture was concentrated in vacuo. The obtained amino acid was used in next step without further purification.

To a solution of above amino acid in dry $\mathrm{MeOH}(4.0 \mathrm{~mL})$ was added $\mathrm{NaBH}_{4}(23.0$ $\mathrm{mg}, 0.610 \mathrm{mmol}$ ) and stirred at room temperature for $20 \mathrm{~min}$. The reaction was quenched with $1 \mathrm{~N} \mathrm{HCl}$ to $\mathrm{pH} 2-3$ carefully and stirred for additional $2 \mathrm{~min}$. After being adjusted to $\mathrm{pH}$ 6-7 with aqueous $\mathrm{NaHCO}_{3}$ solution the mixture was extracted with ethyl acetate, washed with brine and dried over sodium sulfate. After concentration, $2 \mathrm{~mL}$ of dry toluene was added to the residue quickly, then solvent was removed by evaporation; this was repeated three times. The obtained amino acid was used in next step without further purification.

To a solution of 2-chloro-1-methylpyridinium iodide $(0.360 \mathrm{~g}, 1.220 \mathrm{mmol}, 10.0$ equiv) and $\mathrm{Et}_{3} \mathrm{~N}(0.60 \mathrm{~mL}, 3.66 \mathrm{mmol}, 30.0$ equiv) in toluene $(20.0 \mathrm{~mL})$ was added a solution of the above amino acid in THF/toluene $(3 / 2,20.0 \mathrm{~mL})$ via a syringe pump over $15 \mathrm{~h}$. The solid was filtered off after stirring for another $2 \mathrm{~h}$ and the filtrate was concentrated and the residue was purified by column chromatography ( $80 \%$ ethyl acetate/hexanes) to give (+)-kopsiyunnanine $\mathrm{C} 1(\mathbf{1 1})(28.3 \mathrm{mg}, 80 \%, 3 \mathrm{steps}) .[\alpha]_{D}^{20}$ $+244.1\left(\mathrm{c} 0.07, \mathrm{CHCl}_{3}\right)\left\{\right.$ ref. $\left.^{[3]}[\alpha]_{D}^{24}-289.6\left(\mathrm{c} 0.07, \mathrm{CHCl}_{3}\right)\right\} .{ }^{1} \mathbf{H}$ NMR $(400 \mathrm{MHz}$, $\left.\mathrm{CDCl}_{3}\right) \delta 7.40(\mathrm{dd}, J=7.2,1.6 \mathrm{~Hz}, 1 \mathrm{H}), 7.35-7.28(\mathrm{~m}, 2 \mathrm{H}), 7.19(\mathrm{~d}, J=7.6 \mathrm{~Hz}, 1 \mathrm{H})$, $6.65(\mathrm{~s}, 1 \mathrm{H}), 5.75(\mathrm{~s}, 1 \mathrm{H}), 4.30(\mathrm{~s}, 2 \mathrm{H}), 4.07(\mathrm{dd}, J=12.4,4.8 \mathrm{~Hz}, 1 \mathrm{H}), 3.68(\mathrm{td}, J=$ $12.4,4.8 \mathrm{~Hz}, 1 \mathrm{H}), 3.28(\mathrm{~s}, 3 \mathrm{H}), 2.41(\mathrm{td}, J=16.8,4.0 \mathrm{~Hz}, 2 \mathrm{H}), 2.22-2.17(\mathrm{~m}, 1 \mathrm{H})$, 1.98-1.88 (m, $2 \mathrm{H}), 1.69$ (td, $J=13.2,3.2 \mathrm{~Hz}, 1 \mathrm{H}), 1.53-1.42(\mathrm{~m}, 3 \mathrm{H}), 1.27-1.20(\mathrm{~m}$, $1 \mathrm{H}), 0.69(\mathrm{t}, J=7.6 \mathrm{~Hz}, 3 \mathrm{H}) .{ }^{13} \mathbf{C} \mathbf{N M R}\left(100 \mathrm{MHz}, \mathrm{CDCl}_{3}\right) \delta 177.3,140.2,137.9$, 132.0, 131.4, 128.0, 127.2, 126.8, 126.3, 116.4, 111.7, 66.0, 57.0, 43.2, 39.0, 36.6, 32.5, 30.1, 28.1, 19.0, 8.2. HRMS (ESI) calcd for $\mathrm{C}_{21} \mathrm{H}_{27} \mathrm{O}_{2} \mathrm{~N}_{2}[\mathrm{M}+\mathrm{H}]^{+}$339.2073, found 339.2077 . 

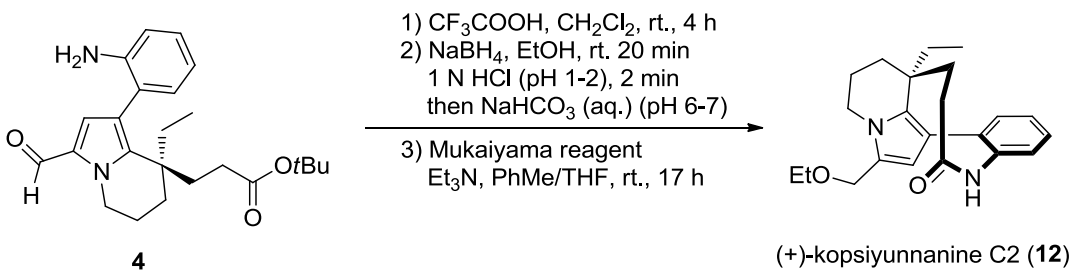

Trifluoroacetic acid $(1.0 \mathrm{~mL})$ was added to the solution of the ester 4 (40.0 $\mathrm{mg}$, $0.120 \mathrm{mmol})$ in dichloromethane $(2.0 \mathrm{~mL})$ dropwise. After being stirred at room temperature for $4 \mathrm{~h}$, the mixture was concentrated in vacuo. The obtained amino acid was used in next step without further purification.

To a solution of above amino acid in dry EtOH $(4.0 \mathrm{~mL})$ was added $\mathrm{NaBH}_{4}(23.0$ $\mathrm{mg}, 0.610 \mathrm{mmol}$ ) and stirred at room temperature for $20 \mathrm{~min}$. The reaction was quenched with $1 \mathrm{~N} \mathrm{HCl}$ to $\mathrm{pH} 2-3$ carefully and stirred for additional $2 \mathrm{~min}$. After being adjusted to $\mathrm{pH}$ 6-7 with aqueous $\mathrm{NaHCO}_{3}$ solution the mixture was extracted with ethyl acetate, washed with brine and dried over sodium sulfate. After concentration, $2 \mathrm{~mL}$ of dry toluene was added to the residue quickly, then solvent was removed by evaporation; this was repeated three times. The obtained amino acid was used in next step without further purification.

To a solution of 2-chloro-1-methylpyridinium iodide $(0.360 \mathrm{~g}, 1.22 \mathrm{mmol}, 10.0$ equiv) and $\mathrm{Et}_{3} \mathrm{~N}(0.60 \mathrm{~mL}, 3.66 \mathrm{mmol}, 30.0$ equiv) in toluene $(20.0 \mathrm{~mL})$ was added a solution of the above amino acid in $\mathrm{THF} /$ toluene $(3 / 2,20.0 \mathrm{~mL})$ via a syringe pump over $15 \mathrm{~h}$. The solid was filtered off after stirring for another $2 \mathrm{~h}$ and the filtrate was concentrated and the residue was purified by column chromatography (70\% ethyl acetate/hexanes) to give (+)-kopsiyunnanine C2 (12) (24.9 mg, 70\%, 3 steps). $[\alpha]_{D}^{20}$ $+138.5\left(\mathrm{c} 0.08, \mathrm{CHCl}_{3}\right)\left\{\right.$ ref. $\left.^{[3]}[\alpha]_{D}^{25}-163.1\left(\mathrm{c} 0.06, \mathrm{CHCl}_{3}\right)\right\} .{ }^{1} \mathbf{H}$ NMR $(400 \mathrm{MHz}$, $\left.\mathrm{CDCl}_{3}\right) \delta 7.39(\mathrm{dd}, J=7.2,2.0 \mathrm{~Hz}, 1 \mathrm{H}), 7.35-7.28(\mathrm{~m}, 2 \mathrm{H}), 7.19(\mathrm{dd}, J=7.6,1.2 \mathrm{~Hz}$, $1 \mathrm{H}), 6.64$ (s, $1 \mathrm{H}), 5.73$ (s, $1 \mathrm{H}), 4.34$ (s, $2 \mathrm{H}), 4.07$ (dd, J = 12.0, $4.8 \mathrm{~Hz}, 1 \mathrm{H}), 3.69$ (td, $J=12.4,5.2 \mathrm{~Hz}, 1 \mathrm{H}), 3.47(\mathrm{qd}, J=6.8,0.8 \mathrm{~Hz}, 2 \mathrm{H}), 2.41(\mathrm{td}, J=28.8,12.6 \mathrm{~Hz}$, $2 \mathrm{H}), 2.23-2.15(\mathrm{~m}, 1 \mathrm{H}), 1.98-1.91(\mathrm{~m}, 2 \mathrm{H}), 1.67(\mathrm{dt}, J=13.6,6.8 \mathrm{~Hz}, 1 \mathrm{H})$, 1.54-1.42 (m, 3 H), 1.27-1.24 (m, $1 \mathrm{H}), 1.20$ (t, $J=7.0 \mathrm{~Hz}, 3 \mathrm{H}), 0.69$ (t, $J=7.4 \mathrm{~Hz}, 3$ H). ${ }^{13} \mathrm{C}$ NMR $\left(100 \mathrm{MHz}, \mathrm{CDCl}_{3}\right) \delta 177.3,140.3,137.8,131.9,131.5,127.9,127.2$, 126.8, 126.6, 116.3, 111.4, 64.9, 64.3, 43.2, 39.0, 36.5, 32.4, 30.1, 28.1, 19.0, 15.2, 8.1. HRMS (ESI) calcd for $\mathrm{C}_{22} \mathrm{H}_{29} \mathrm{O}_{2} \mathrm{~N}_{2}[\mathrm{M}+\mathrm{H}]^{+} 353.2229$, found 353.2221. 


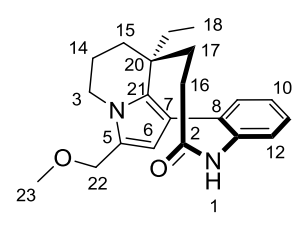

(+)-kopsiyunnanine C1 (11)

\begin{tabular}{|c|c|c|c|c|c|c|}
\hline \multicolumn{7}{|c|}{ Kopsiyunnanine C1 } \\
\hline \multirow[b]{2}{*}{ Atom \# } & \multicolumn{3}{|c|}{${ }^{1} \mathrm{H}$ NMR } & \multicolumn{3}{|c|}{${ }^{13} \mathrm{C}$ NMR } \\
\hline & $\begin{array}{l}\text { Natural } \\
(\delta)\end{array}$ & $\begin{array}{c}\text { Synthetic } \\
(\delta)\end{array}$ & $\Delta \delta$ & $\begin{array}{l}\text { Natural } \\
(\delta)\end{array}$ & $\begin{array}{c}\text { Synthetic } \\
(\delta)\end{array}$ & $\Delta \delta$ \\
\hline 1 & 6.6 & 6.7 & 0.1 & - & - & - \\
\hline 2 & - & - & - & 177.3 & 177.3 & 0.0 \\
\hline \multirow{2}{*}{3} & 4.1 & 4.1 & 0 & \multirow{2}{*}{43.3} & \multirow{2}{*}{43.2} & \multirow{2}{*}{-0.1} \\
\hline & 3.7 & 3.7 & 0 & & & \\
\hline 5 & - & - & - & 126.3 & 126.3 & 0.0 \\
\hline 6 & 5.8 & 5.8 & 0.0 & 111.8 & 111.7 & -0.1 \\
\hline 7 & - & - & - & 116.5 & 116.4 & -0.1 \\
\hline 8 & - & - & - & 140.3 & 140.2 & -0.1 \\
\hline 9 & 7.4 & 7.4 & 0.0 & 131.5 & 131.4 & -0.1 \\
\hline 10 & 7.3 & 7.3 & 0.0 & 127.3 & 127.2 & -0.1 \\
\hline 11 & 7.3 & 7.3 & 0.0 & 128.1 & 128.0 & -0.1 \\
\hline 12 & 7.2 & 7.2 & 0.0 & 126.9 & 126.8 & -0.1 \\
\hline 13 & - & - & - & 137.9 & 137.9 & 0.0 \\
\hline \multirow{2}{*}{14} & 2.3 & 2.2 & -0.1 & \multirow{2}{*}{19.1} & \multirow{2}{*}{19.0} & \multirow{2}{*}{-0.1} \\
\hline & 1.9 & 1.9 & 0.0 & & & \\
\hline \multirow[b]{2}{*}{15} & 1.7 & 1.7 & 0.0 & \multirow[b]{2}{*}{32.5} & \multirow[b]{2}{*}{32.5} & \multirow[b]{2}{*}{0.0} \\
\hline & 1.5 & $\begin{array}{c}1.53-1.42 \\
(\mathrm{~m})\end{array}$ & - & & & \\
\hline \multirow{2}{*}{16} & 2.4 & 2.4 & 0.0 & \multirow{2}{*}{28.2} & \multirow{2}{*}{28.1} & \multirow{2}{*}{-0.1} \\
\hline & 2.0 & 2.0 & 0.0 & & & \\
\hline \multirow[b]{2}{*}{17} & 2.5 & 2.4 & -0.1 & \multirow[b]{2}{*}{36.7} & \multirow[b]{2}{*}{36.6} & \multirow[b]{2}{*}{-0.1} \\
\hline & 1.5 & $\begin{array}{c}1.53-1.42 \\
(\mathrm{~m})\end{array}$ & - & & & \\
\hline 18 & 0.7 & 0.7 & 0.0 & 8.2 & 8.2 & 0.0 \\
\hline \multirow[t]{2}{*}{19} & 1.5 & $\begin{array}{c}1.53-1.42 \\
(\mathrm{~m})\end{array}$ & - & \multirow[t]{2}{*}{30.2} & \multirow[t]{2}{*}{30.1} & \multirow[t]{2}{*}{-0.1} \\
\hline & 1.2 & 1.2 & 0.0 & & & \\
\hline 20 & - & - & - & 39.1 & 39.0 & -0.1 \\
\hline 21 & - & - & - & 132.1 & 132.0 & -0.1 \\
\hline 22 & 4.3 & 4.3 & 0.0 & 66.1 & 66.0 & -0.1 \\
\hline 23 & 3.6 & 3.3 & -0.3 & 57.1 & 57.0 & -0.1 \\
\hline
\end{tabular}




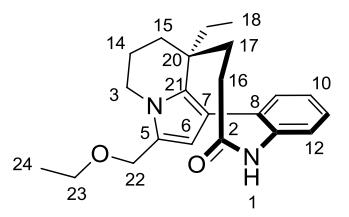

(+)-kopsiyunnanine C2 (12)

\begin{tabular}{|c|c|c|c|c|c|c|}
\hline \multicolumn{7}{|c|}{ Kopsiyunnanine C2 } \\
\hline \multirow[b]{2}{*}{ Atom \# } & \multicolumn{3}{|c|}{${ }^{1} \mathrm{H}$ NMR } & \multicolumn{3}{|c|}{${ }^{13} \mathrm{C}$ NMR } \\
\hline & $\begin{array}{c}\text { Natural } \\
(\delta)\end{array}$ & $\begin{array}{c}\text { Synthetic } \\
(\delta)\end{array}$ & $\Delta \delta$ & $\begin{array}{c}\text { Natural } \\
(\delta)\end{array}$ & 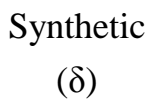 & $\Delta \delta$ \\
\hline 1 & 6.6 & 6.6 & 0.0 & - & - & - \\
\hline 2 & - & - & - & 177.2 & 177.3 & 0.1 \\
\hline \multirow{2}{*}{3} & 4.1 & 4.1 & 0 & \multirow{2}{*}{43.3} & \multirow{2}{*}{43.2} & \multirow{2}{*}{-0.1} \\
\hline & 3.7 & 3.7 & 0 & & & \\
\hline 5 & - & - & - & 126.6 & 126.6 & 0.0 \\
\hline 6 & 5.7 & 5.7 & 0.0 & 111.4 & 111.4 & 0.0 \\
\hline 7 & - & - & - & 116.4 & 116.3 & -0.1 \\
\hline 8 & - & - & - & 140.3 & 140.3 & 0.0 \\
\hline 9 & 7.4 & 7.4 & 0.0 & 131.5 & 131.5 & 0.0 \\
\hline 10 & 7.3 & 7.3 & 0.0 & 127.3 & 127.2 & -0.1 \\
\hline 11 & 7.3 & 7.3 & 0.0 & 128.0 & 127.9 & -0.1 \\
\hline 12 & 7.2 & 7.2 & 0.0 & 126.8 & 126.8 & 0.0 \\
\hline 13 & - & - & - & 137.9 & 137.8 & -0.1 \\
\hline \multirow{2}{*}{14} & 2.2 & 2.2 & 0.0 & \multirow{2}{*}{19.1} & \multirow{2}{*}{19.0} & \multirow{2}{*}{-0.1} \\
\hline & 1.9 & 1.9 & 0.0 & & & \\
\hline \multirow[b]{2}{*}{15} & 1.7 & 1.7 & 0.0 & \multirow[b]{2}{*}{32.5} & \multirow[b]{2}{*}{32.4} & \multirow[b]{2}{*}{-0.1} \\
\hline & 1.5 & $\begin{array}{c}1.54-1.42 \\
(\mathrm{~m})\end{array}$ & - & & & \\
\hline \multirow{2}{*}{16} & 2.4 & 2.4 & 0.0 & \multirow{2}{*}{28.1} & \multirow{2}{*}{28.1} & \multirow{2}{*}{0.0} \\
\hline & 2.0 & 2.0 & 0.0 & & & \\
\hline \multirow[b]{2}{*}{17} & 2.4 & 2.4 & 0.0 & \multirow[b]{2}{*}{36.6} & \multirow[b]{2}{*}{36.5} & \multirow[b]{2}{*}{-0.1} \\
\hline & 1.4 & $\begin{array}{c}1.54-1.42 \\
(\mathrm{~m})\end{array}$ & - & & & \\
\hline 18 & 0.7 & 0.7 & 0.0 & 8.2 & 8.1 & -0.1 \\
\hline \multirow[t]{2}{*}{19} & 1.5 & $\begin{array}{c}1.54-1.42 \\
(\mathrm{~m})\end{array}$ & - & \multirow[t]{2}{*}{30.2} & \multirow[t]{2}{*}{30.1} & \multirow[t]{2}{*}{-0.1} \\
\hline & 1.3 & 1.3 & 0.0 & & & \\
\hline 20 & - & - & - & 39.0 & 39.0 & 0.0 \\
\hline 21 & - & - & - & 131.9 & 131.9 & 0.0 \\
\hline 22 & 4.4 & 4.3 & -0.1 & 64.3 & 64.3 & 0.0 \\
\hline 23 & 3.5 & 3.5 & 0.0 & 64.9 & 64.9 & 0.0 \\
\hline 24 & 1.2 & 1.2 & 0.0 & 15.2 & 15.2 & 0.0 \\
\hline
\end{tabular}




\section{References:}

[1] W.-B. Liu, C. Zheng, C.-X. Zhuo, L.-X. Dai, S.-L. You, J. Am. Chem. Soc., 2012, 134 (10), 4812-4821.

[2] X. Sui, R. Zhu, G. Li, X. Ma, and Z. Gu, J. Am. Chem. Soc., 2012,135(25), 93189321.

[3] Y.-Q. Wu, M. Suehiro, M. Kitajima, T. Matsuzaki, S. Hashimoto, M. Nagaoka, R.-P. Zhang, and H. Takayama, J. Nat. Prod. 2009, 72, 204-209.

[4] M. Banwell, D. Beck, and A. Willis, ARKIVOC, 2006 (iii), 163-174. 


\section{Copies of ${ }^{1} \mathrm{H}$ NMR, ${ }^{13} \mathrm{C}$ NMR and ${ }^{31}$ P NMR spectra}

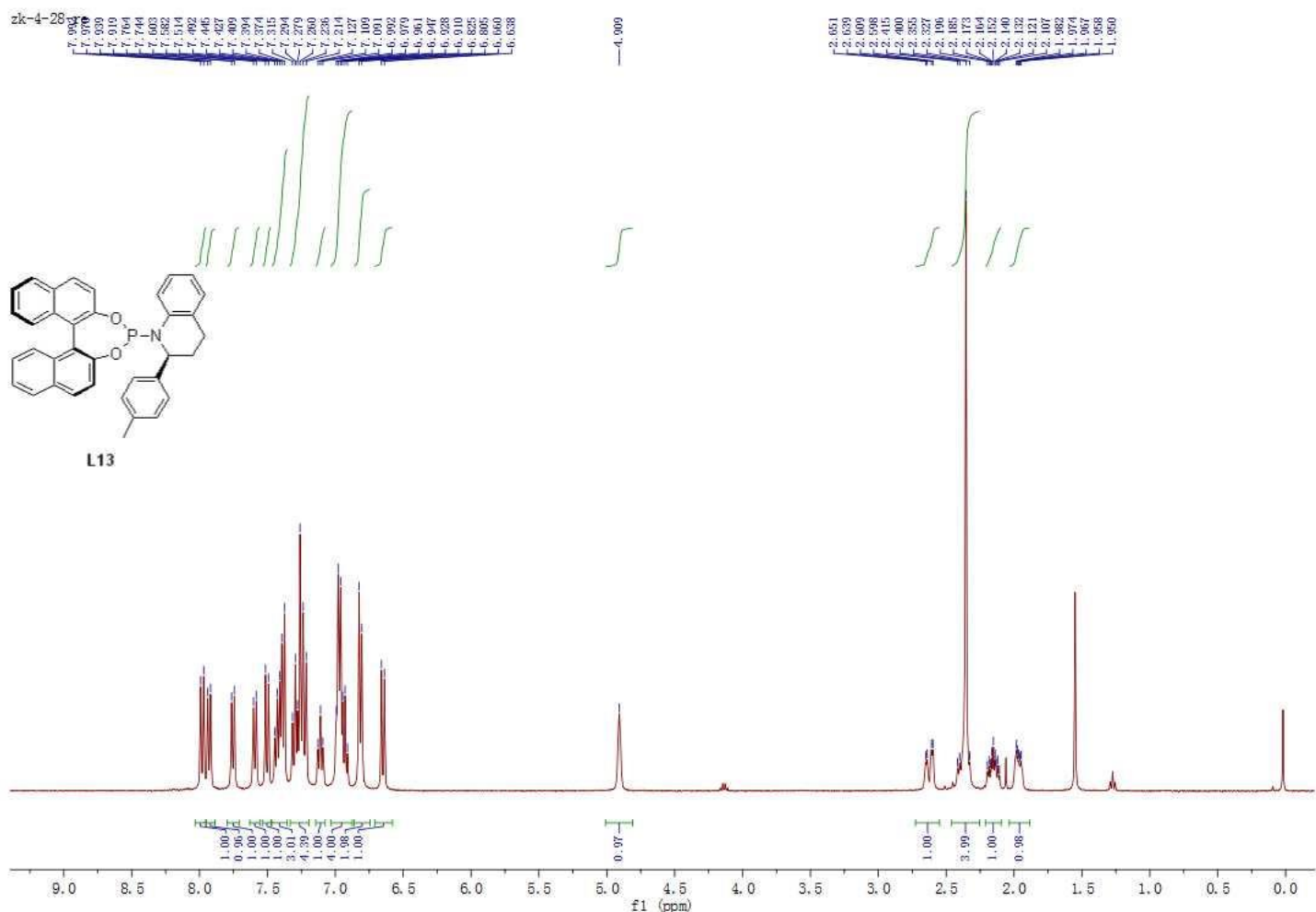

2 leg $4-28-\mathrm{PNMR}$

$\vec{i}$

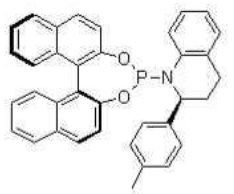

L13

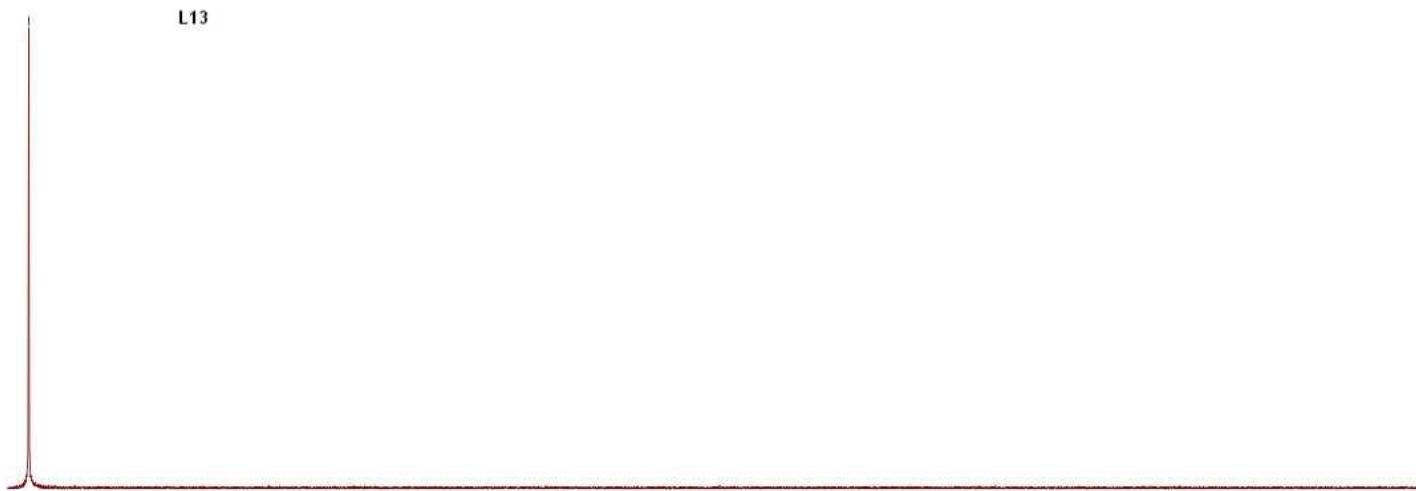

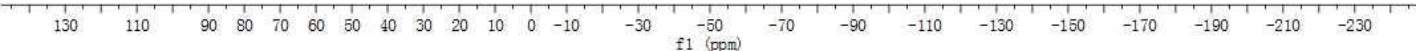



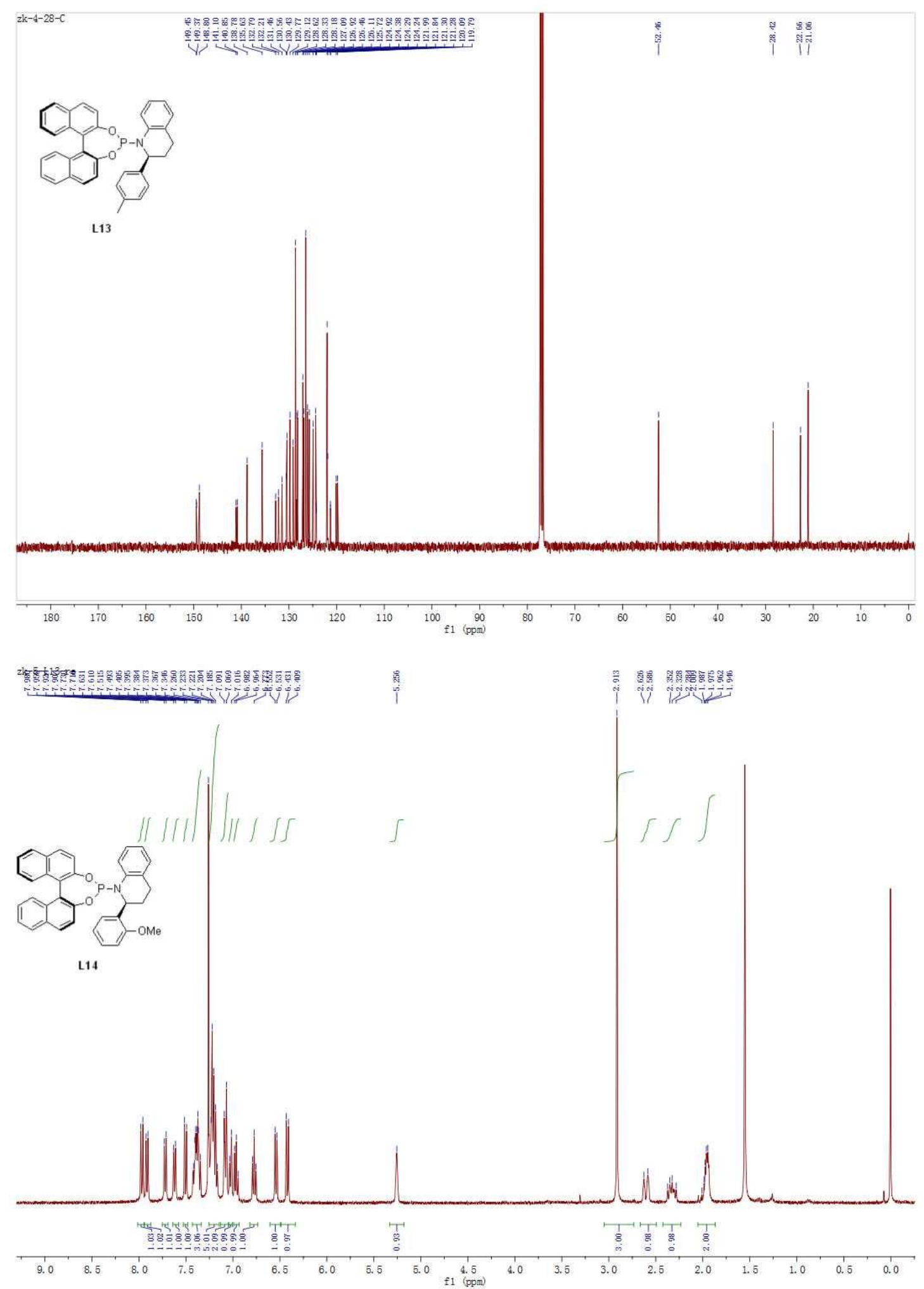

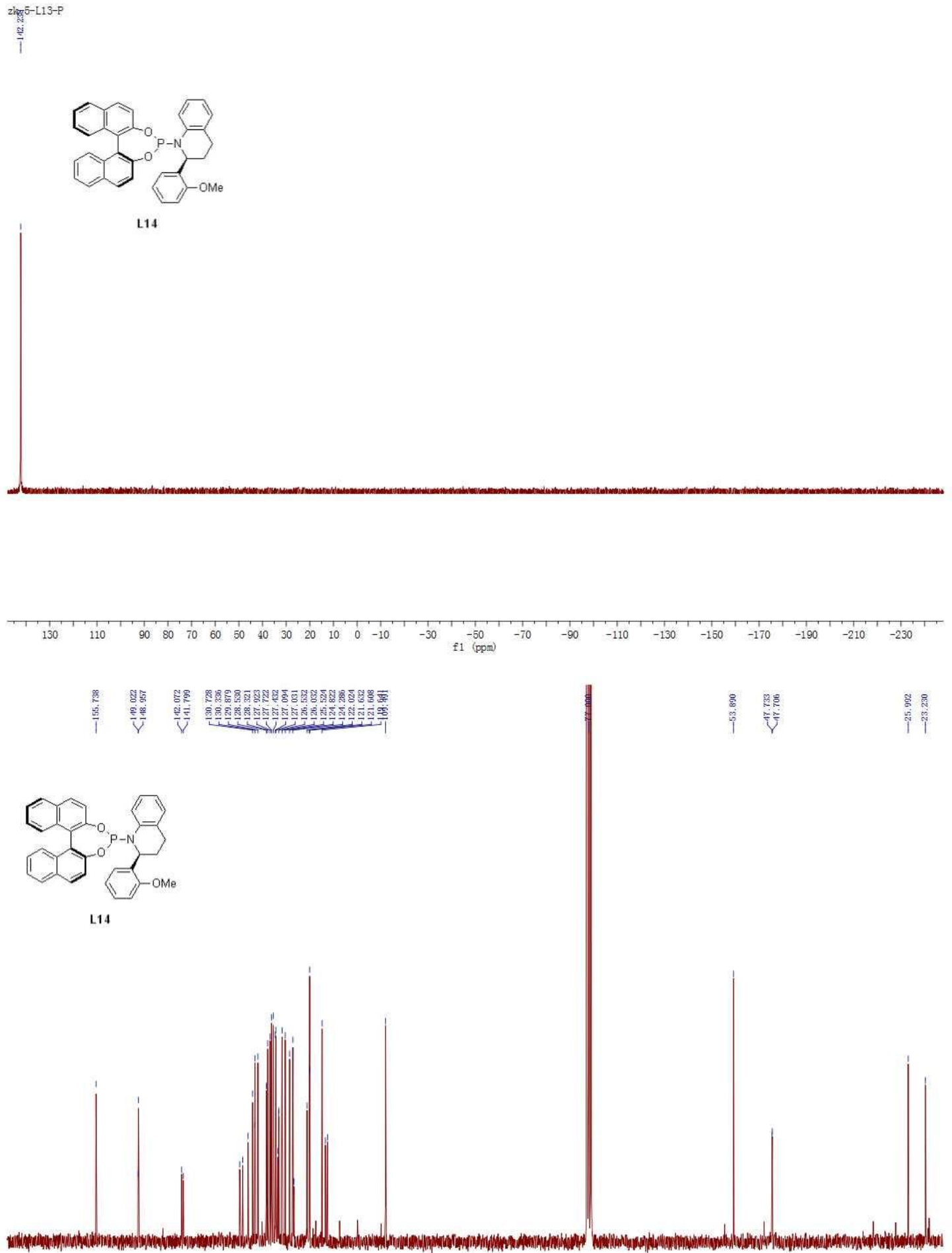

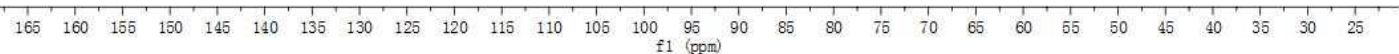




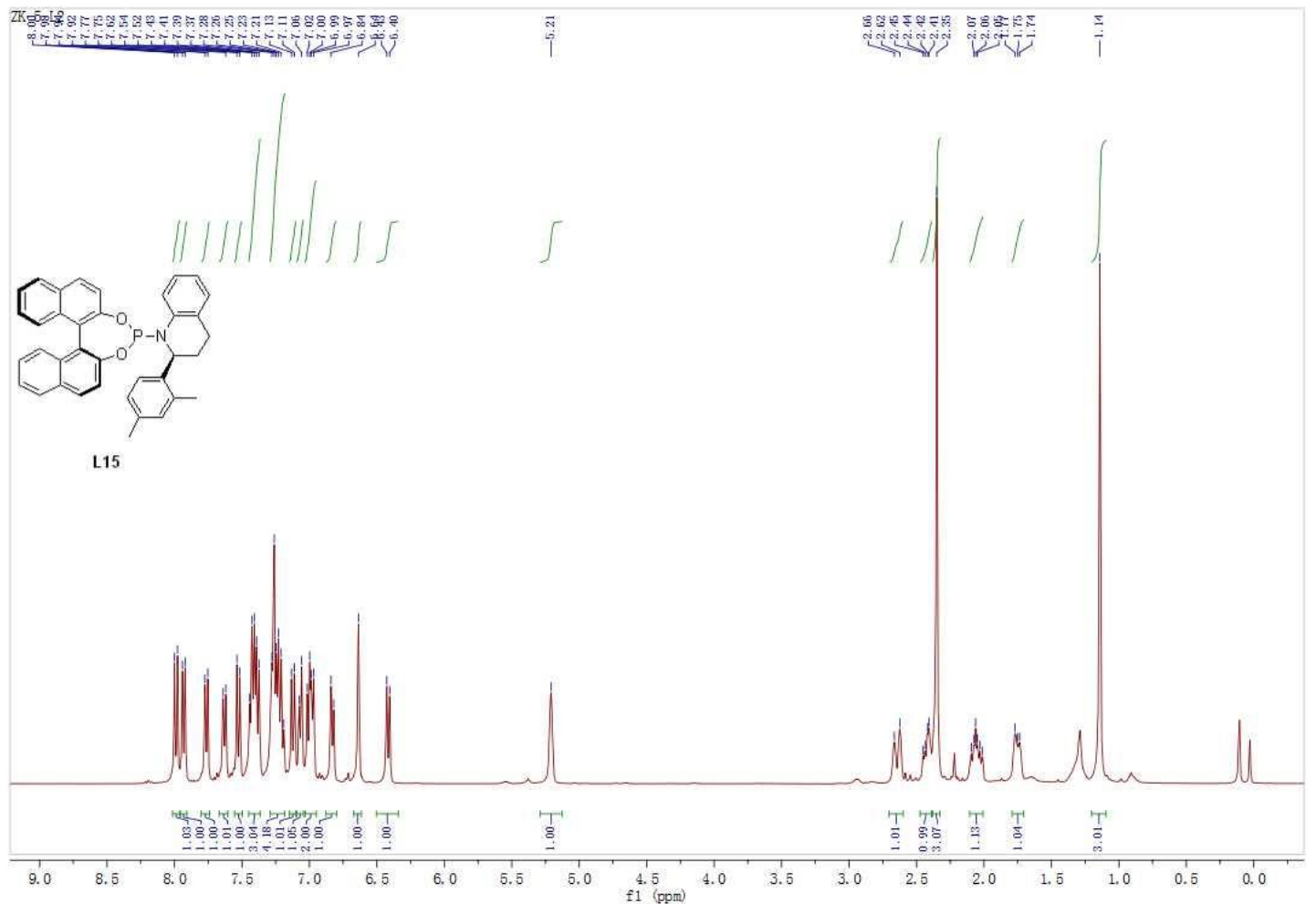

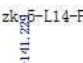

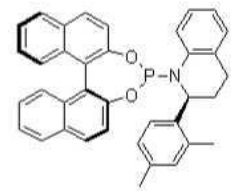

L15

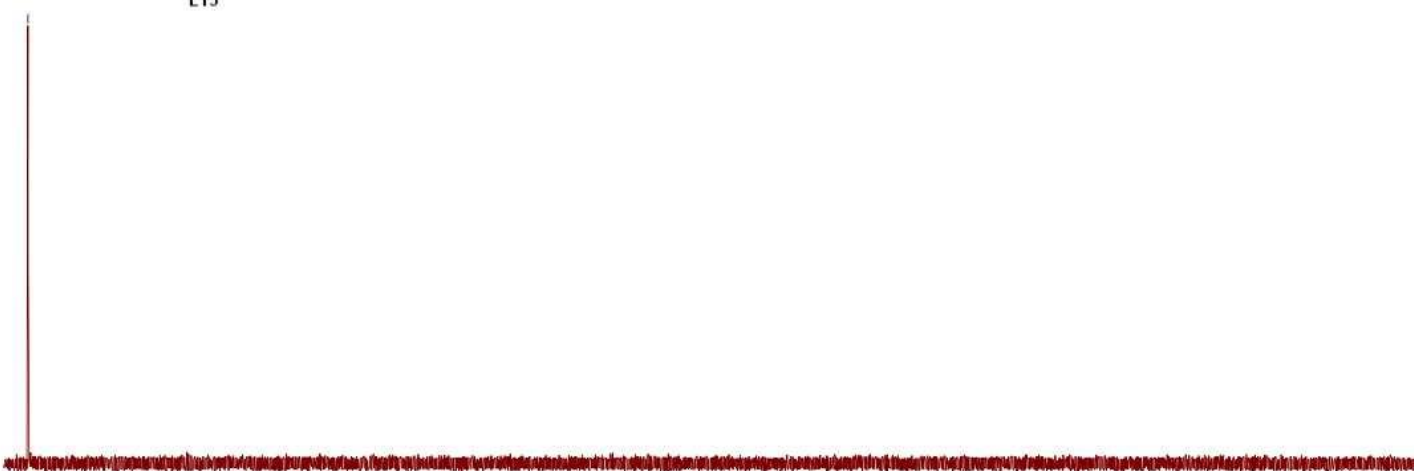

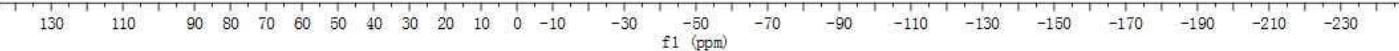



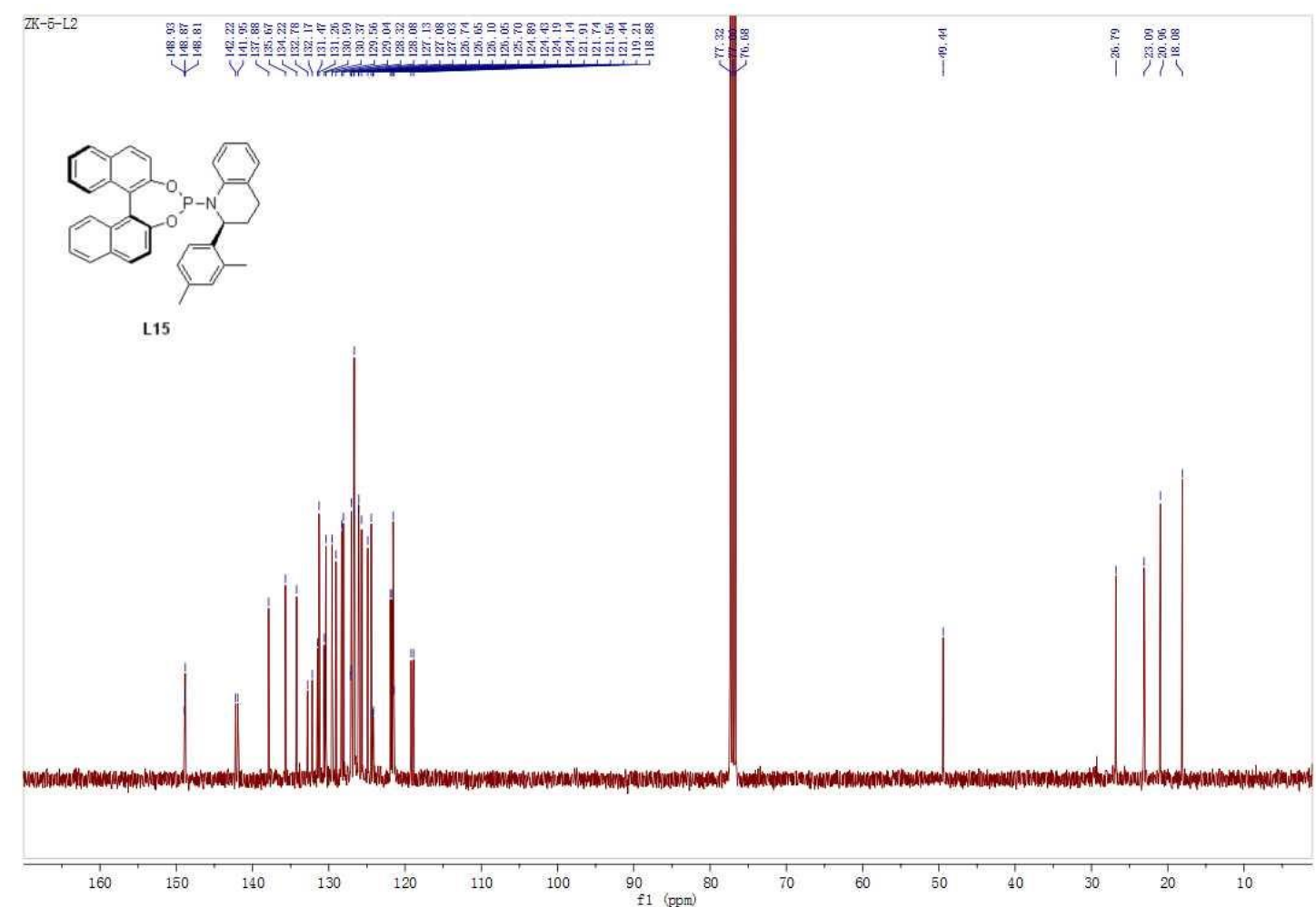

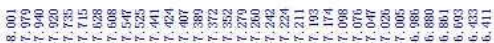

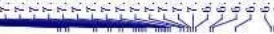

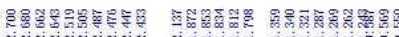
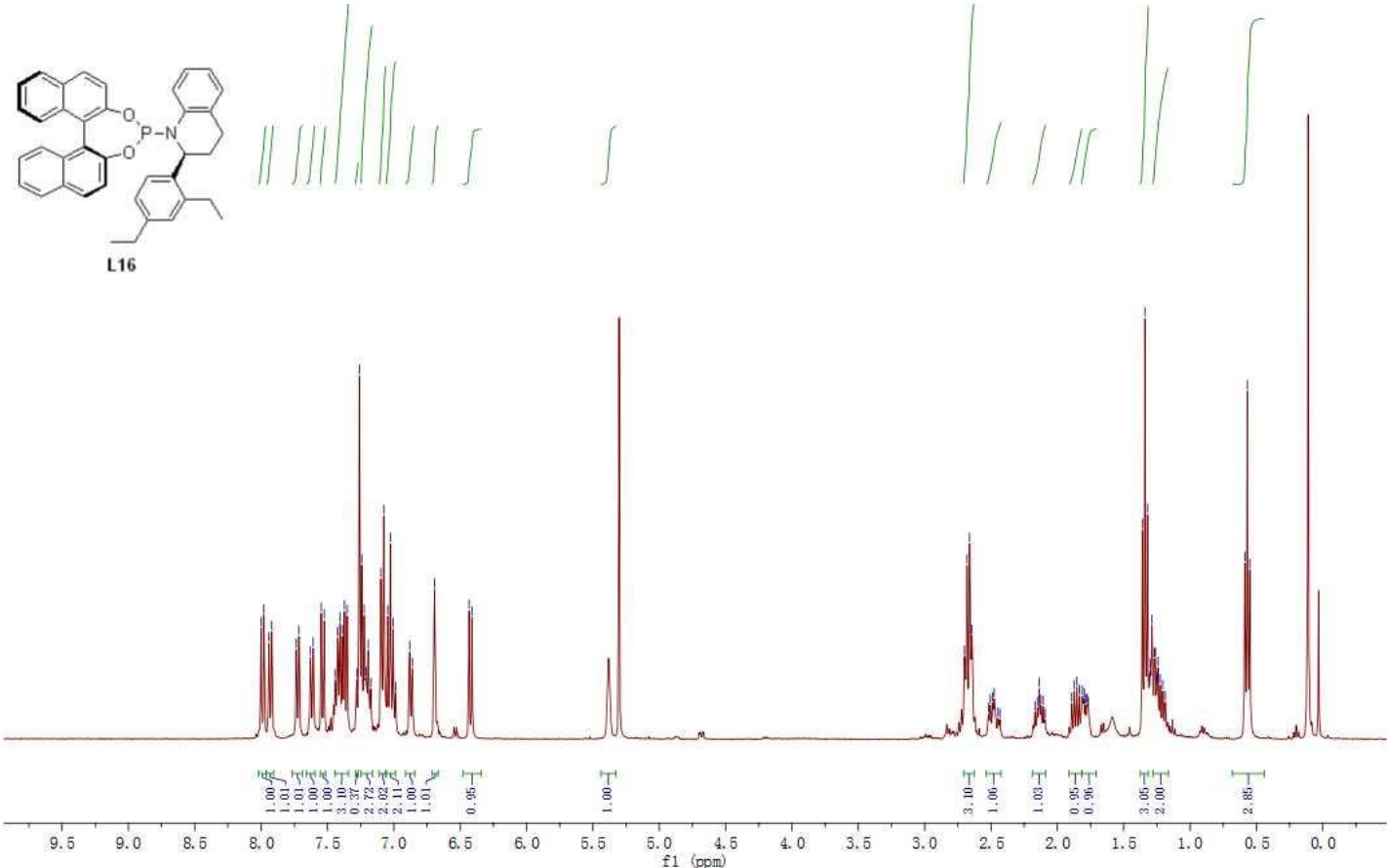

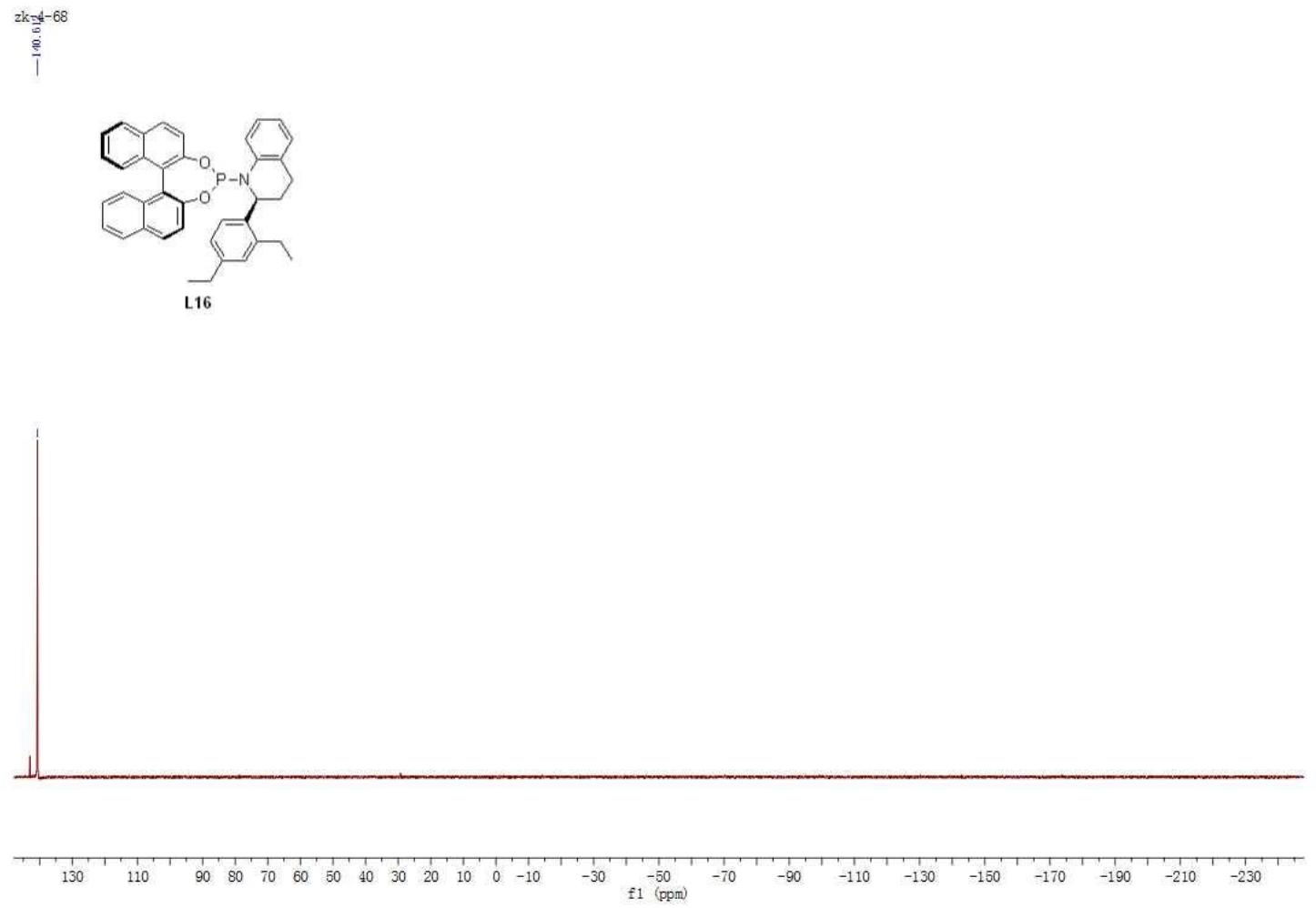

$z k-4-68$

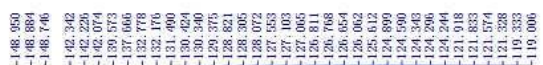

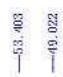

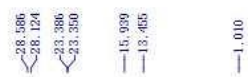
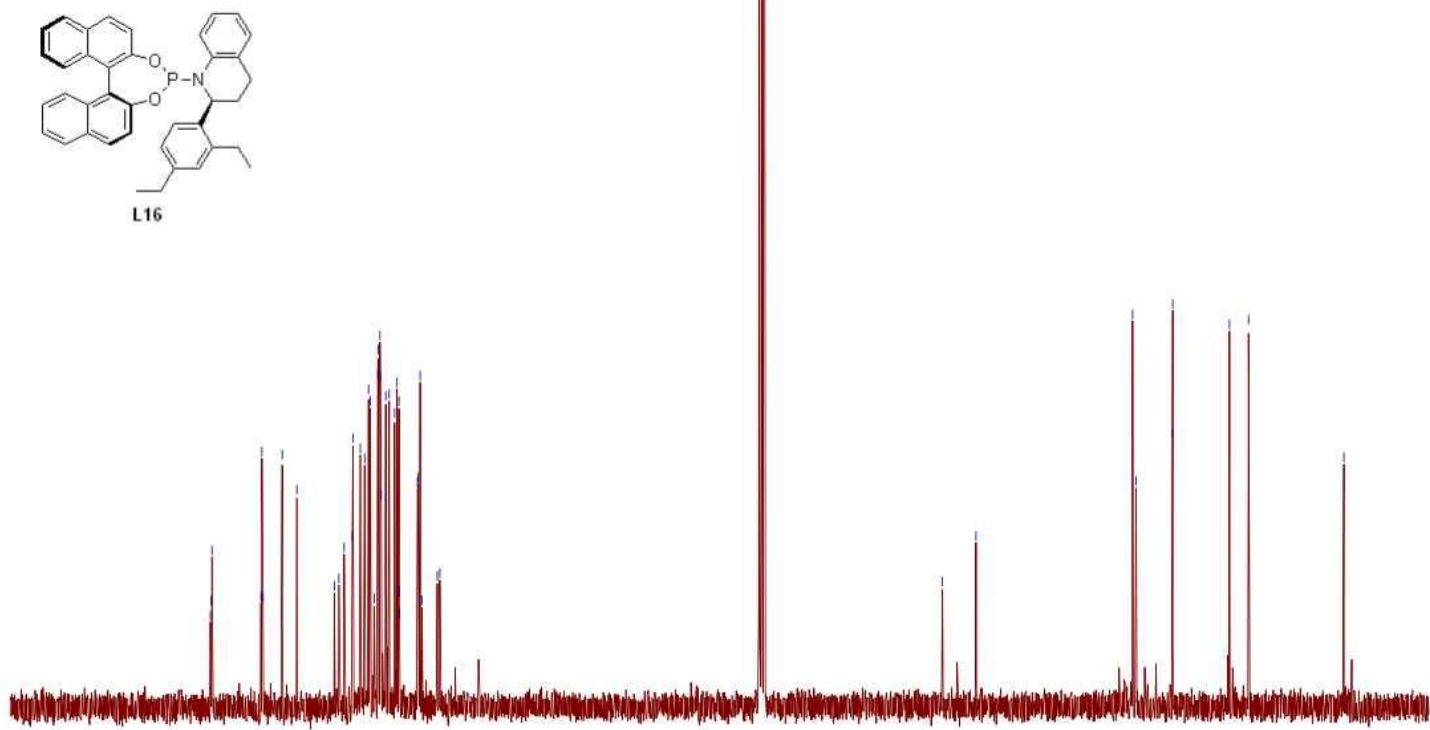

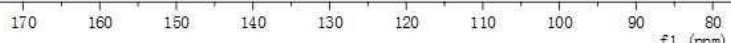

$\begin{array}{lllllllll}1 & 1 & 1 & 1 & 1 & 1 & 1 & 1\end{array}$ 

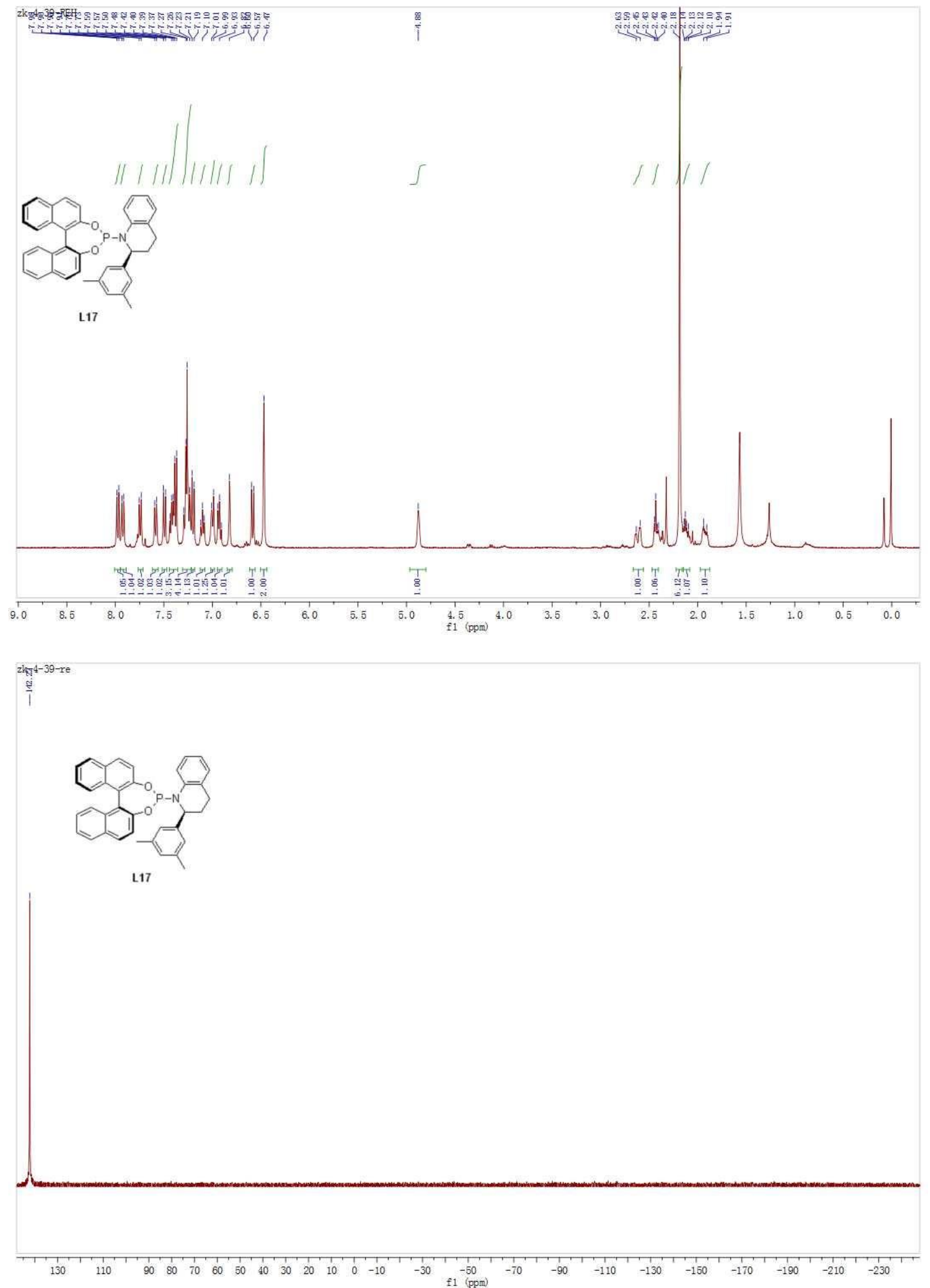

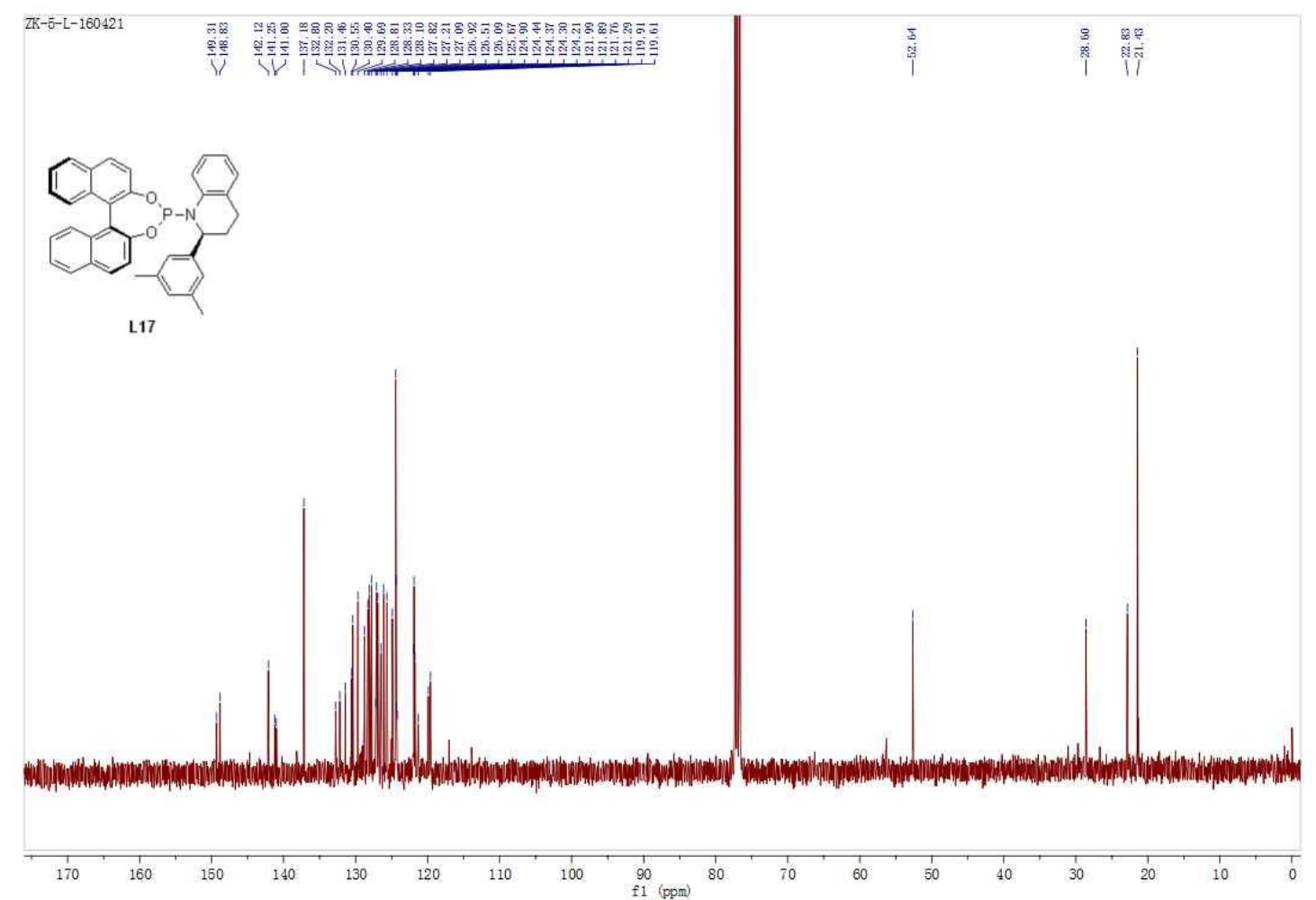

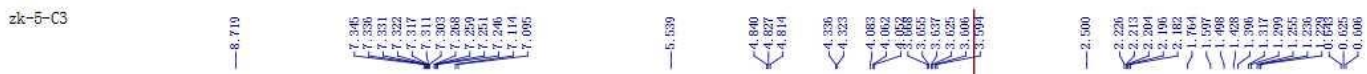

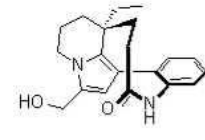

$(+)-k o p s i y u n n a n i n e$
DMSO-d
DMS
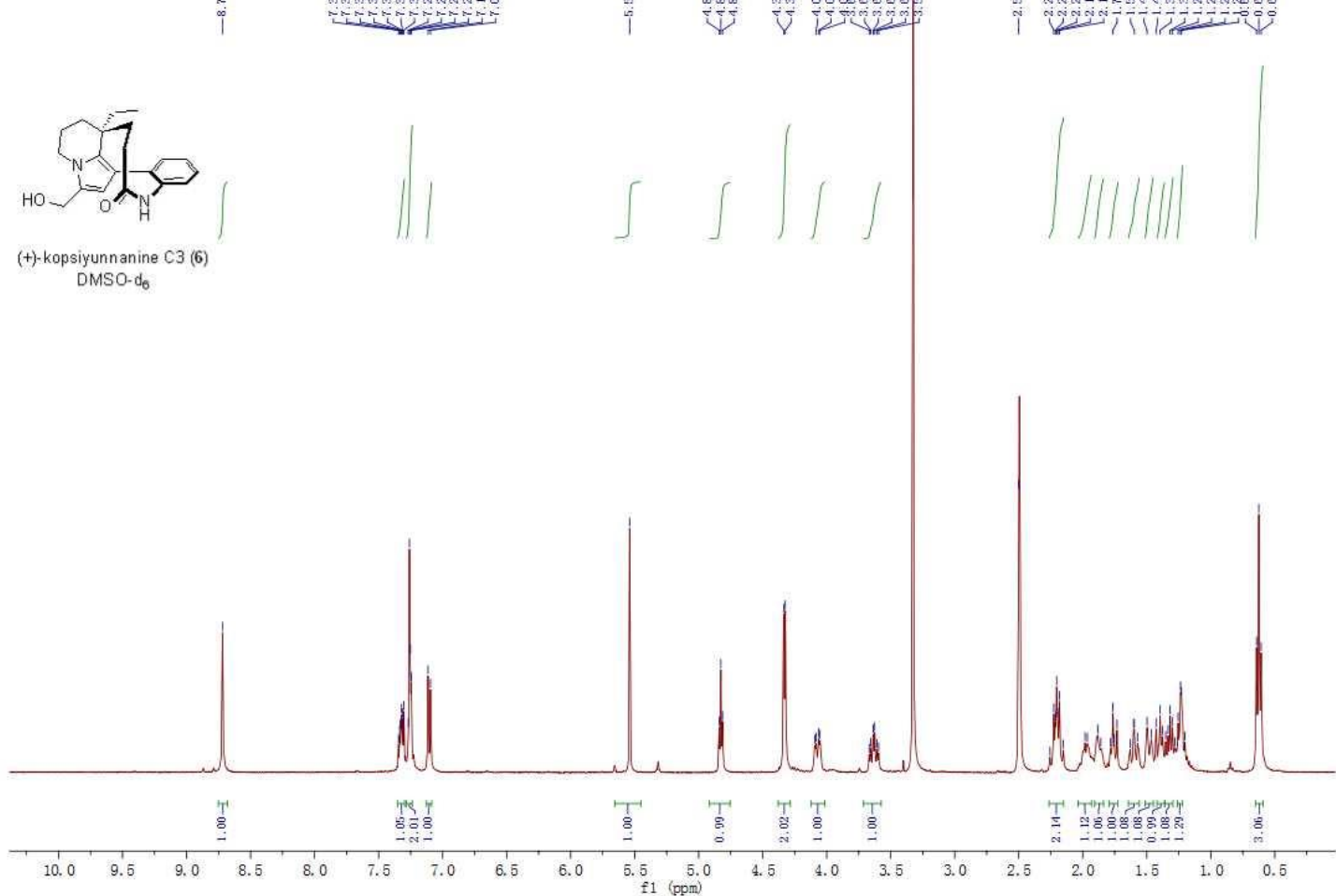

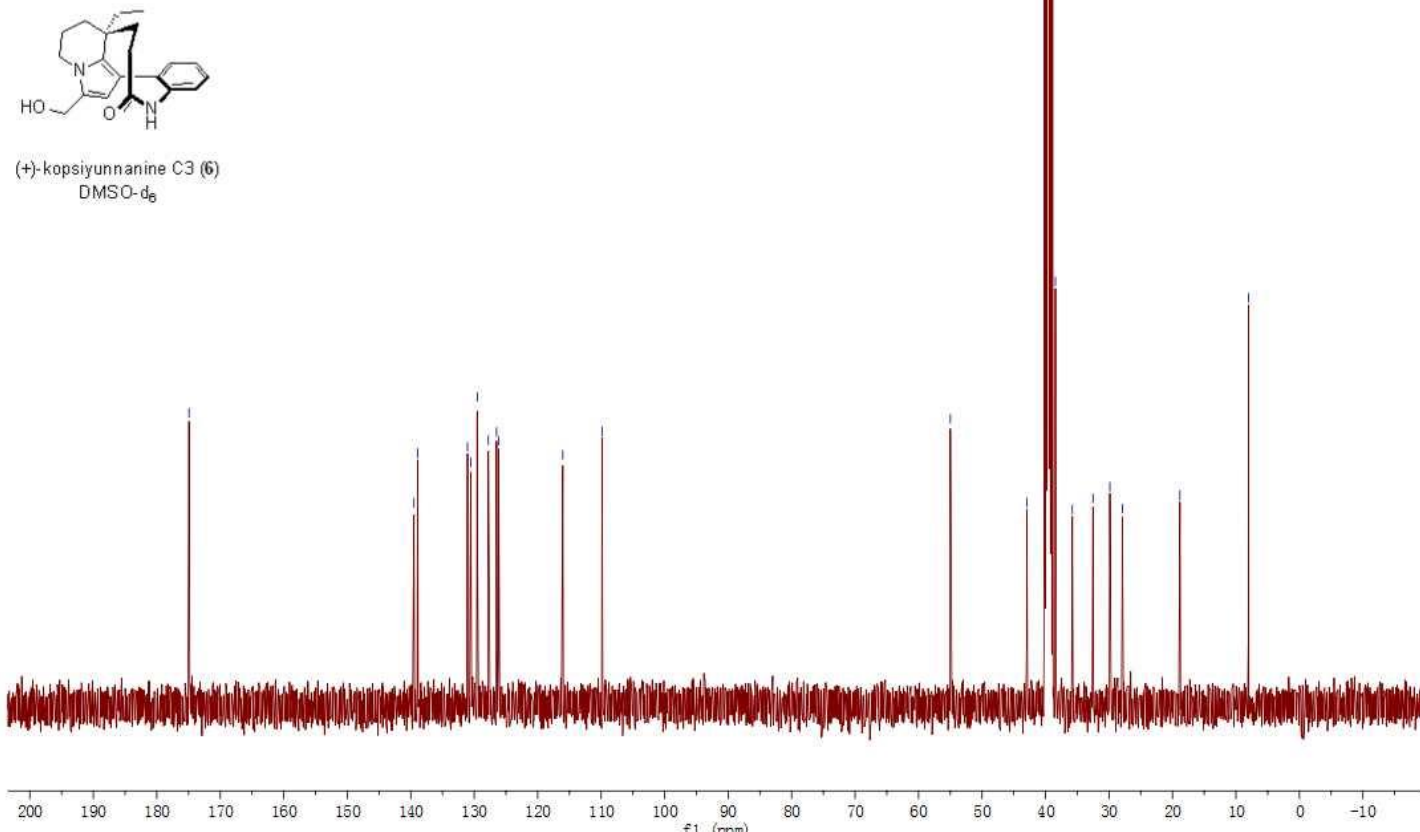

$z k-4-143-c$

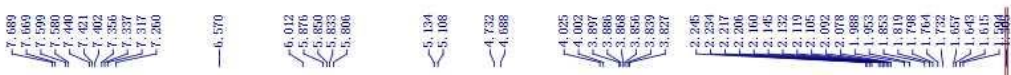<smiles>O=C(CC1CCCc2c(-c3ccccc3[N+](=O)[O-])ccn21)OCc1ccccc1</smiles>

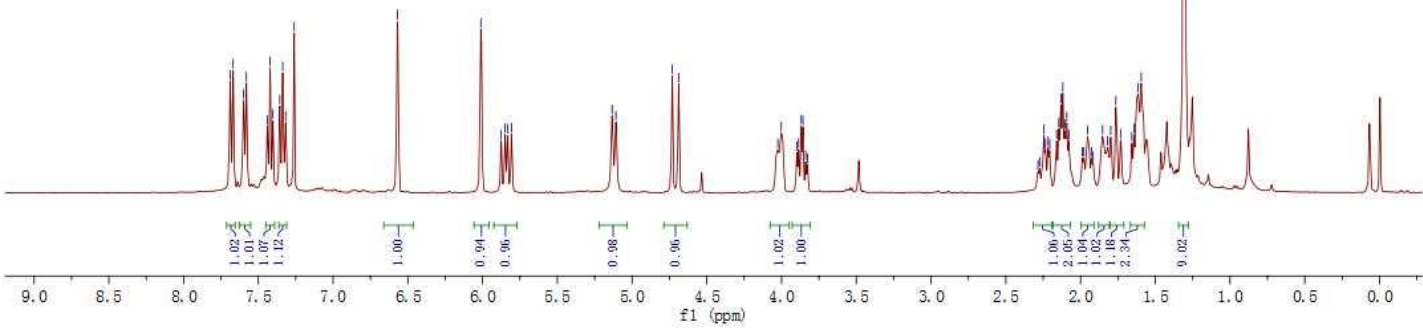



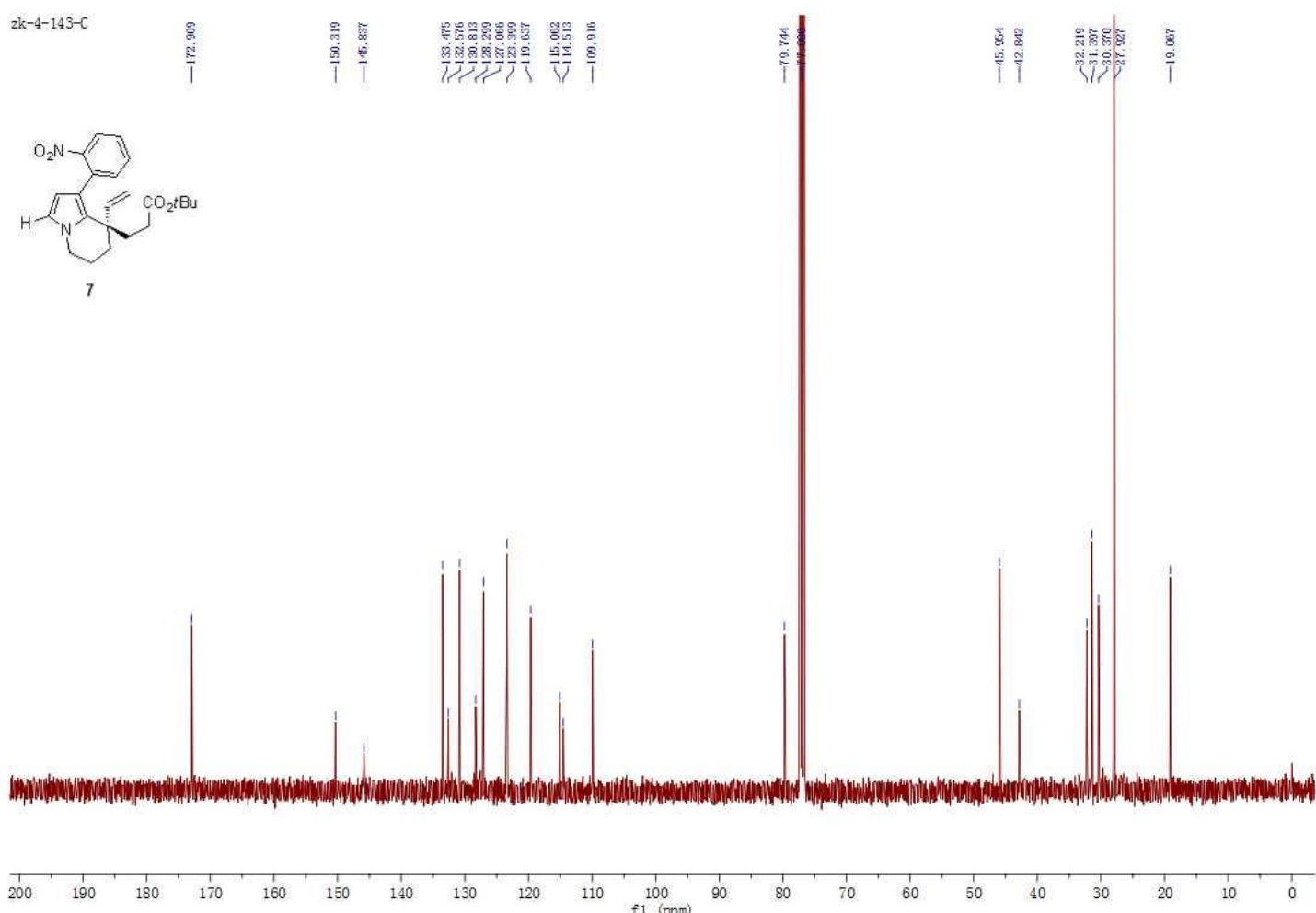

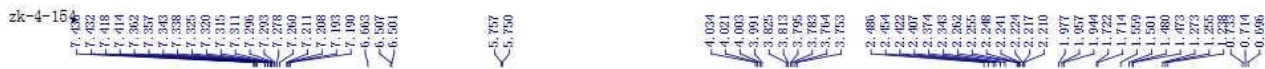

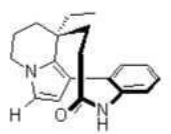

(+)-rhazinilam (8)
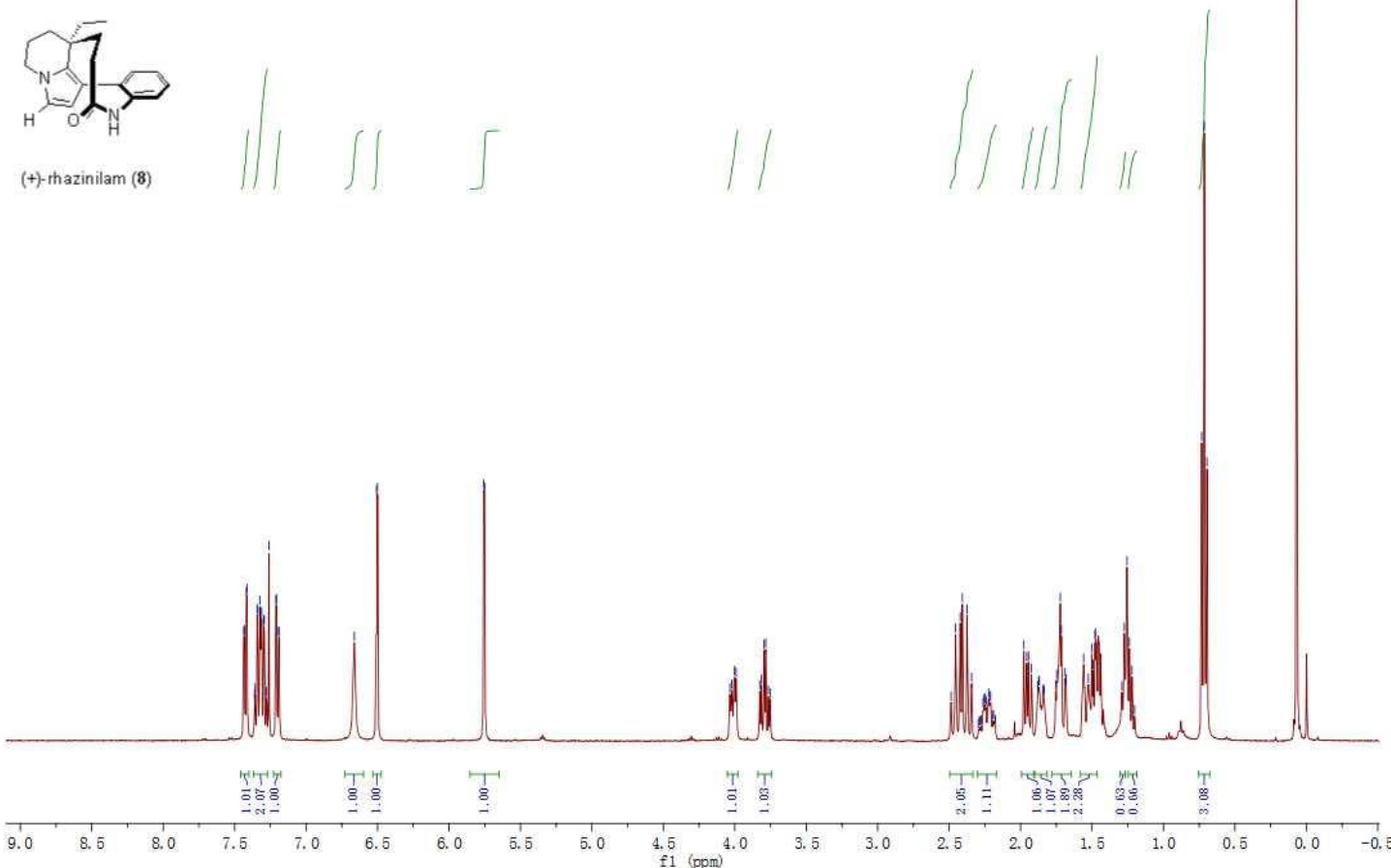

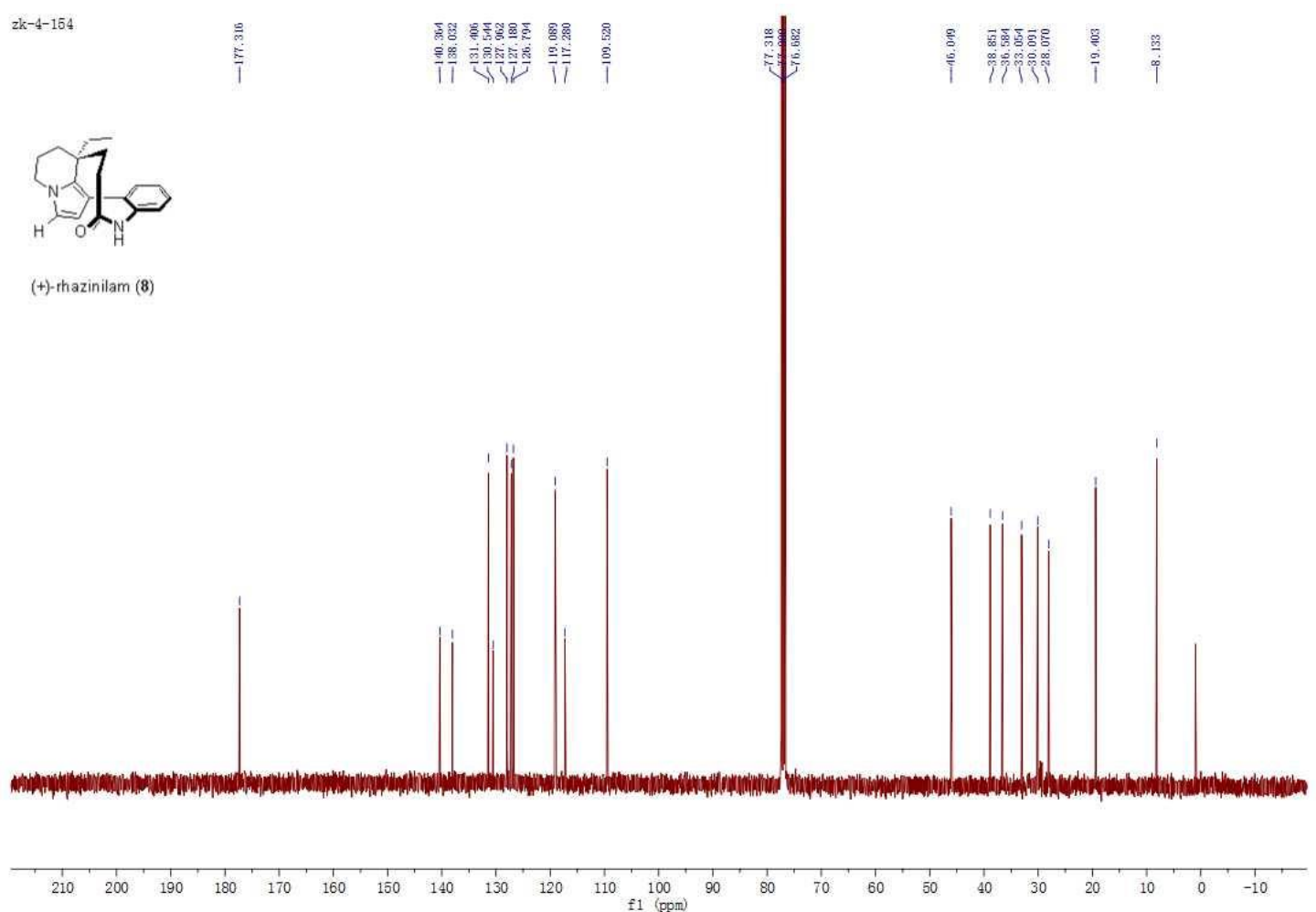

$z k-5-180-p 2$

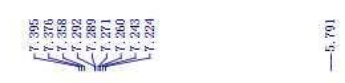

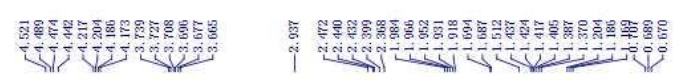
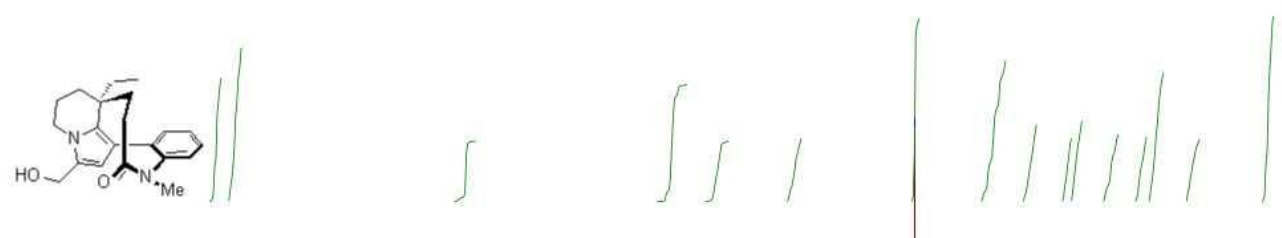

N-Me-6
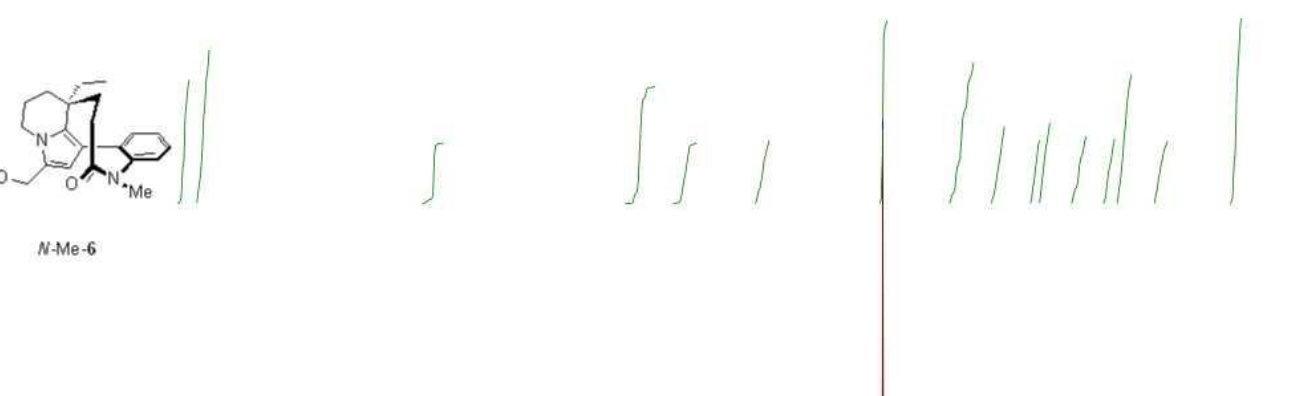

$$
\text { . }
$$

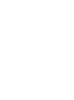



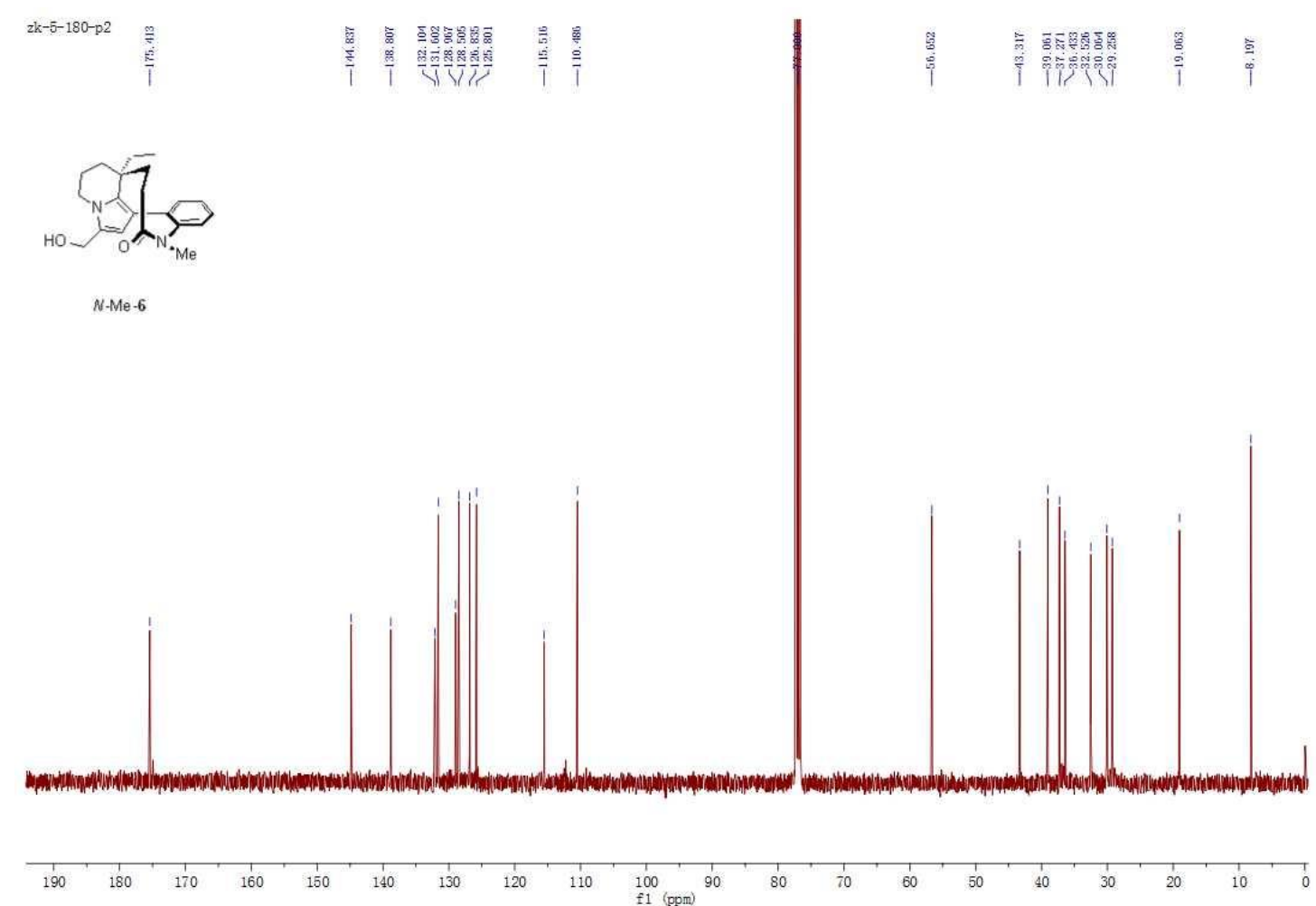

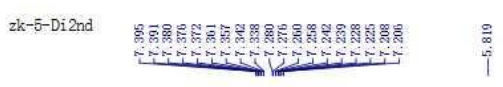

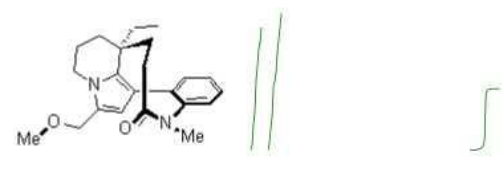

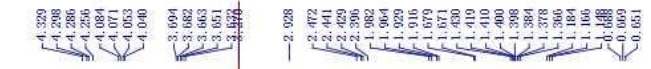

N,OdiMe-6
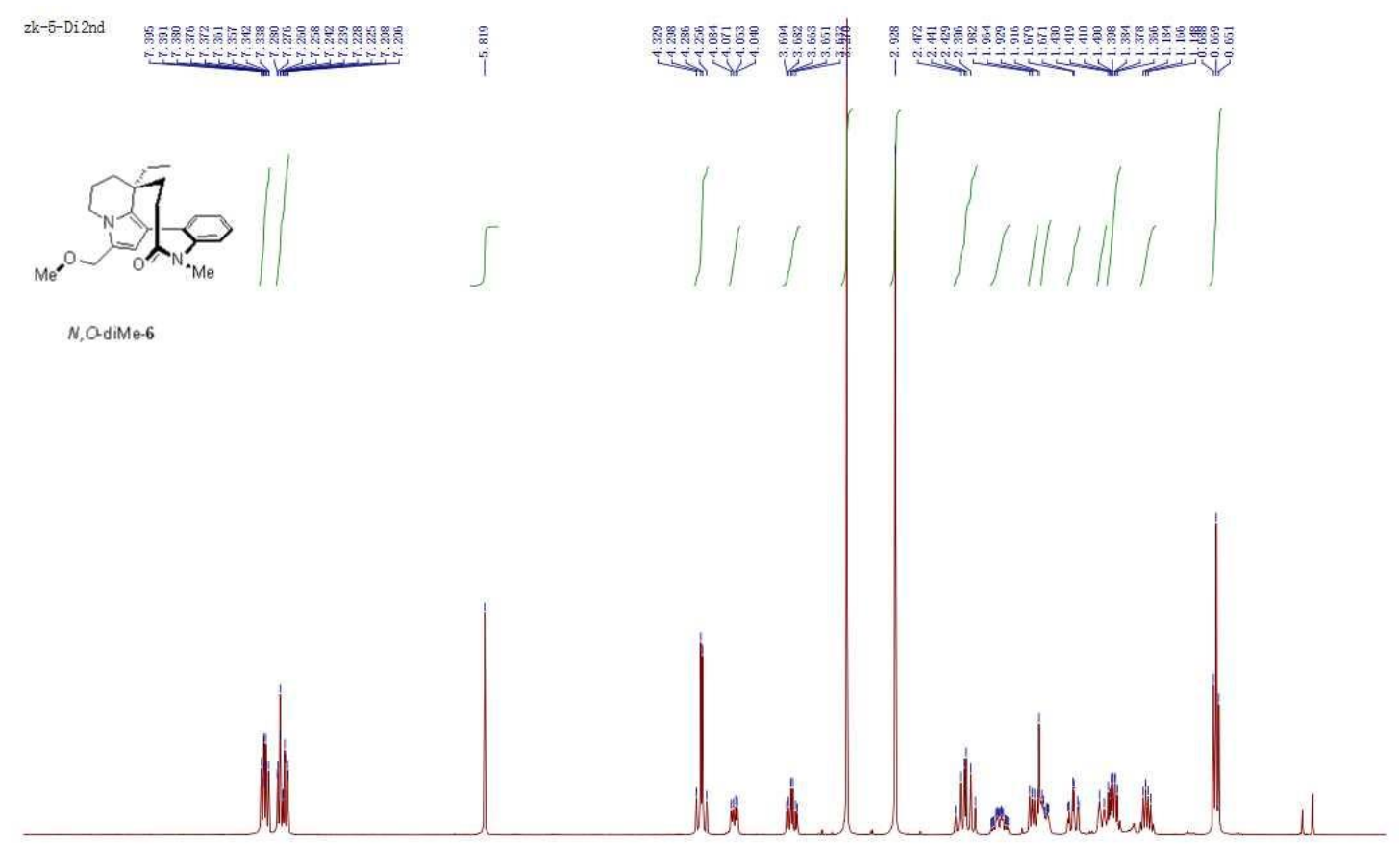

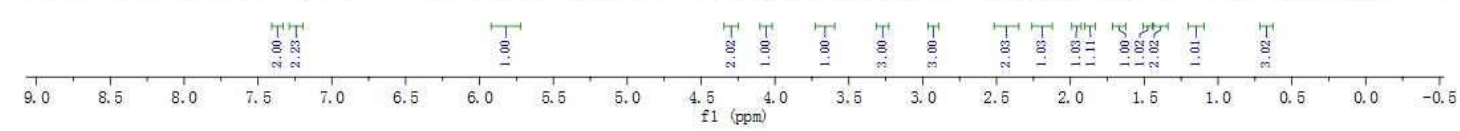



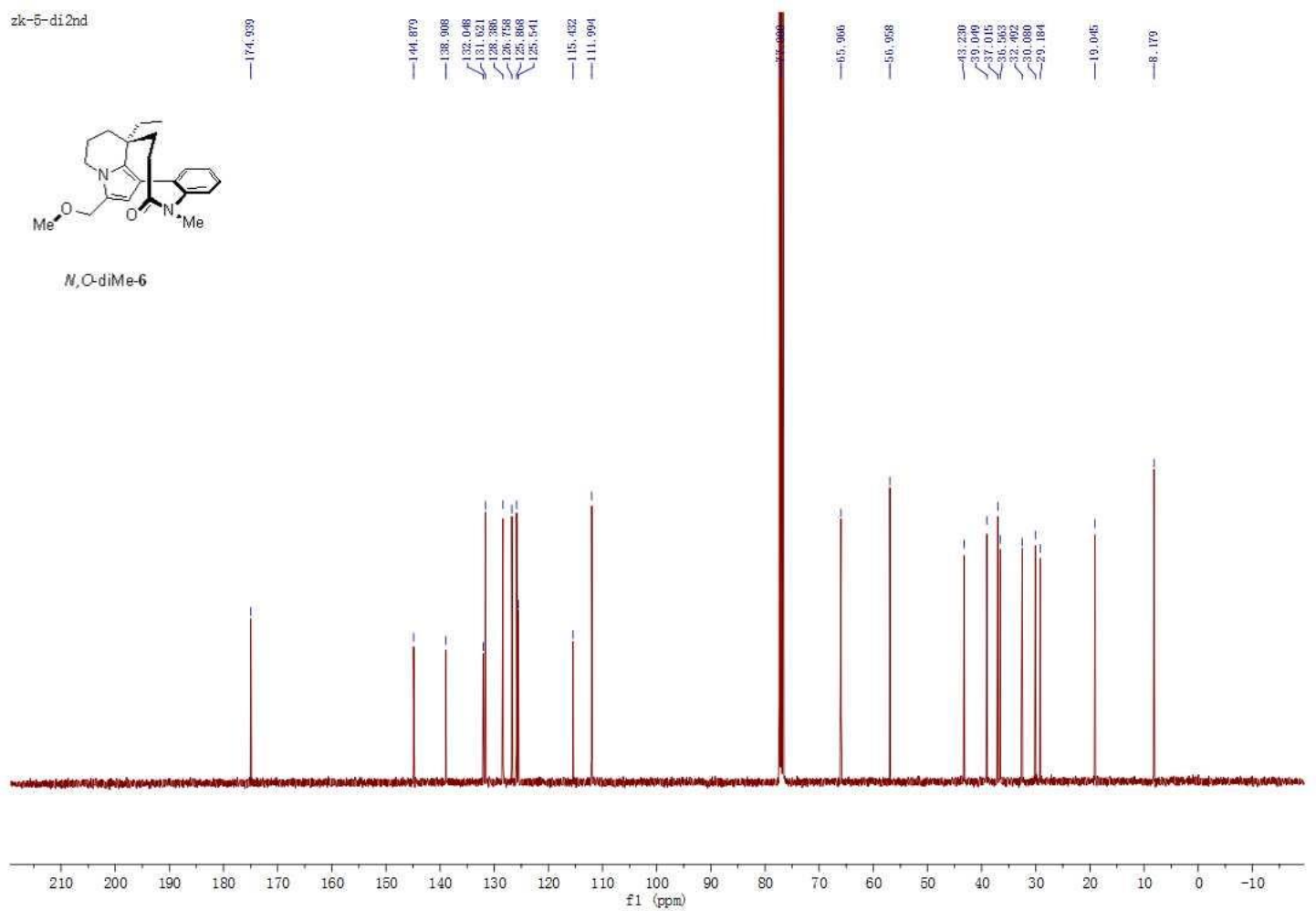

$z k-5-40$

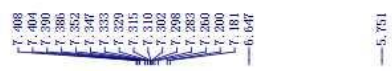

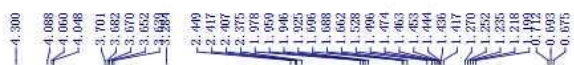

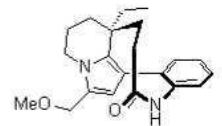
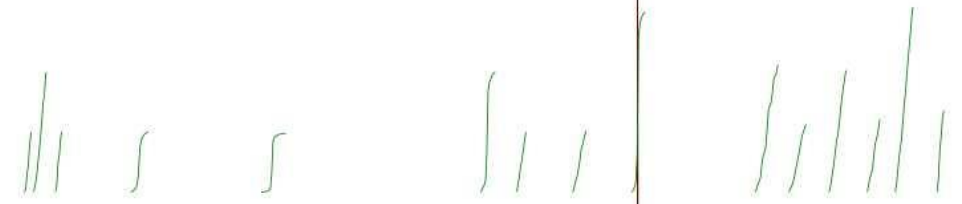

(+)-kopsiyunnanine C1 (11)
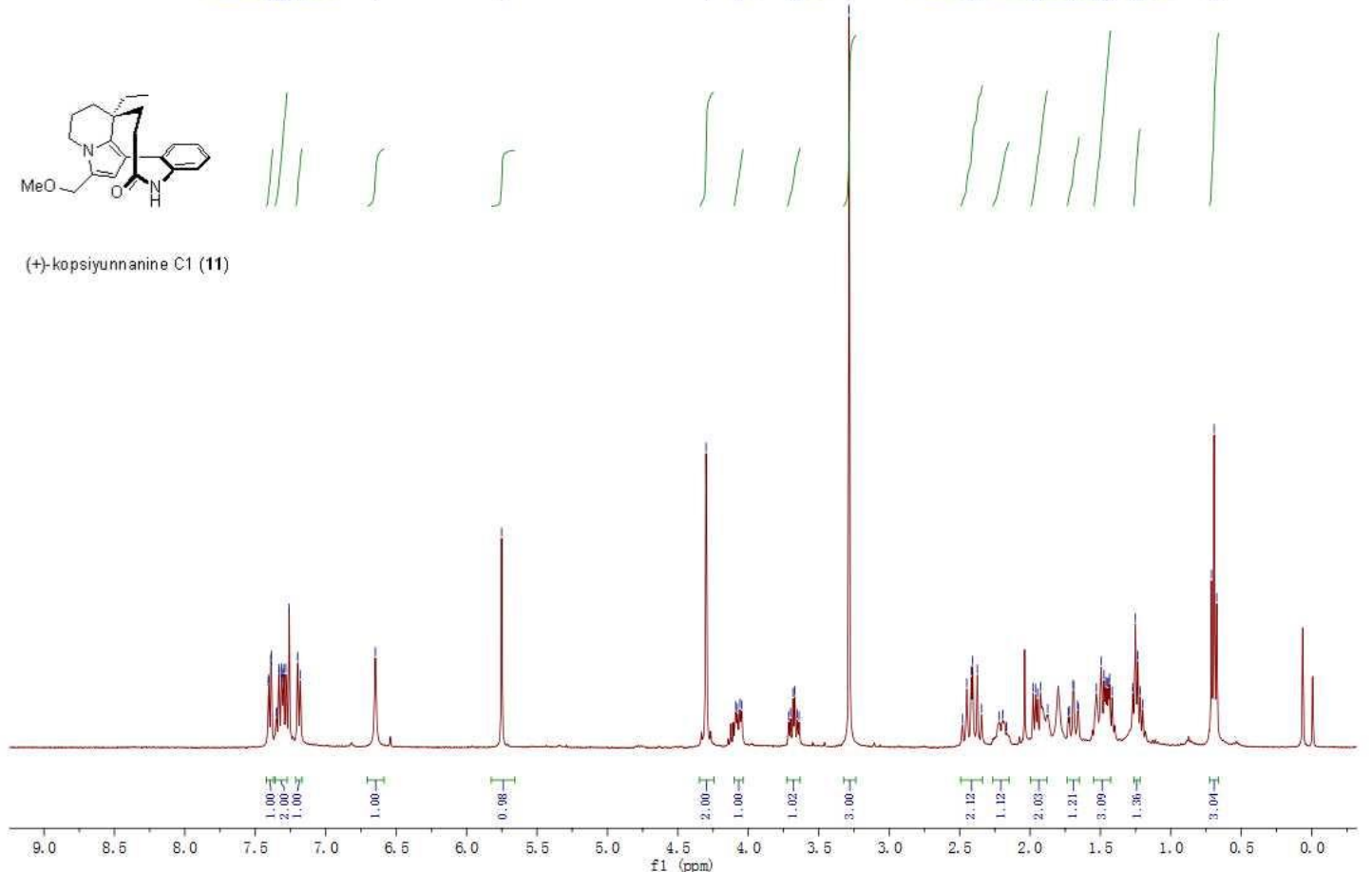

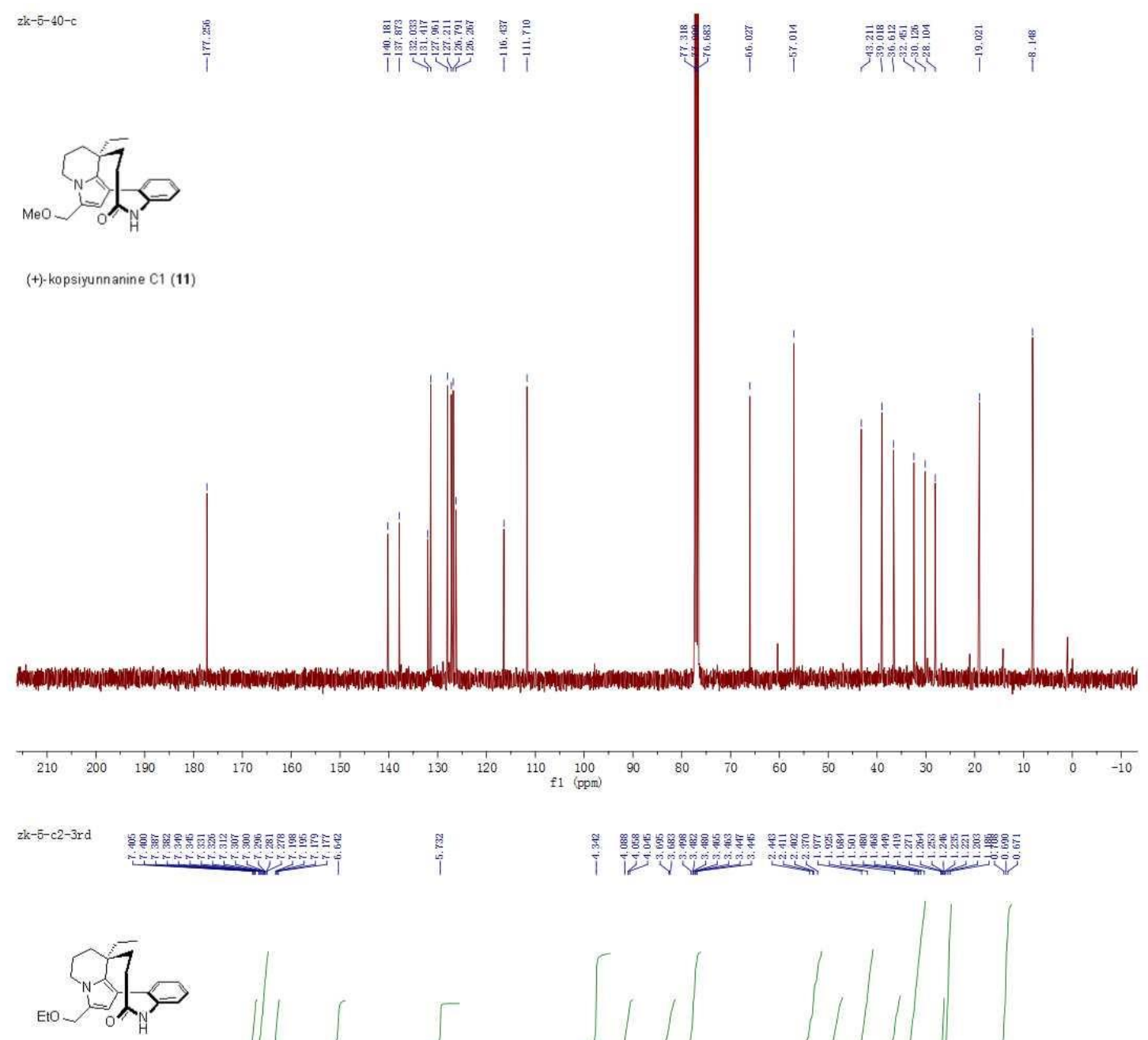

(+)-kopsiyunn anine C2 (12)

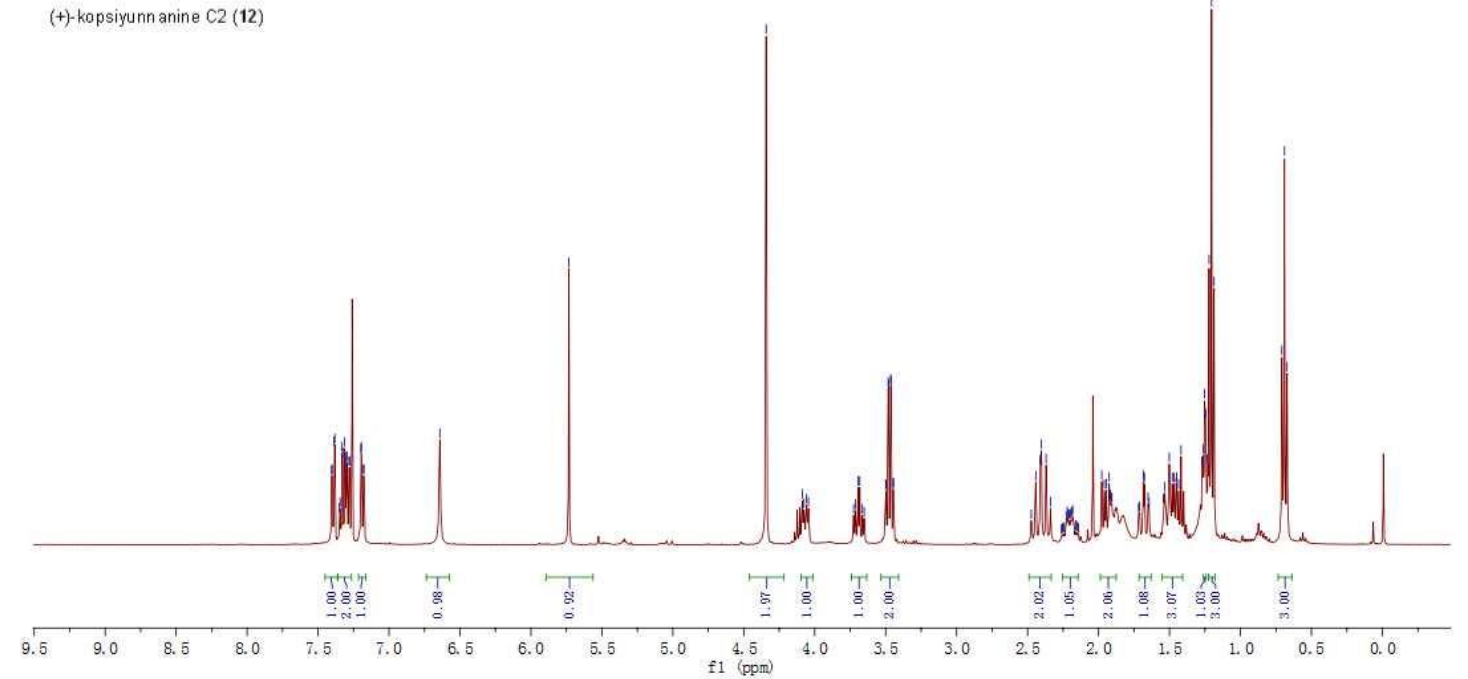



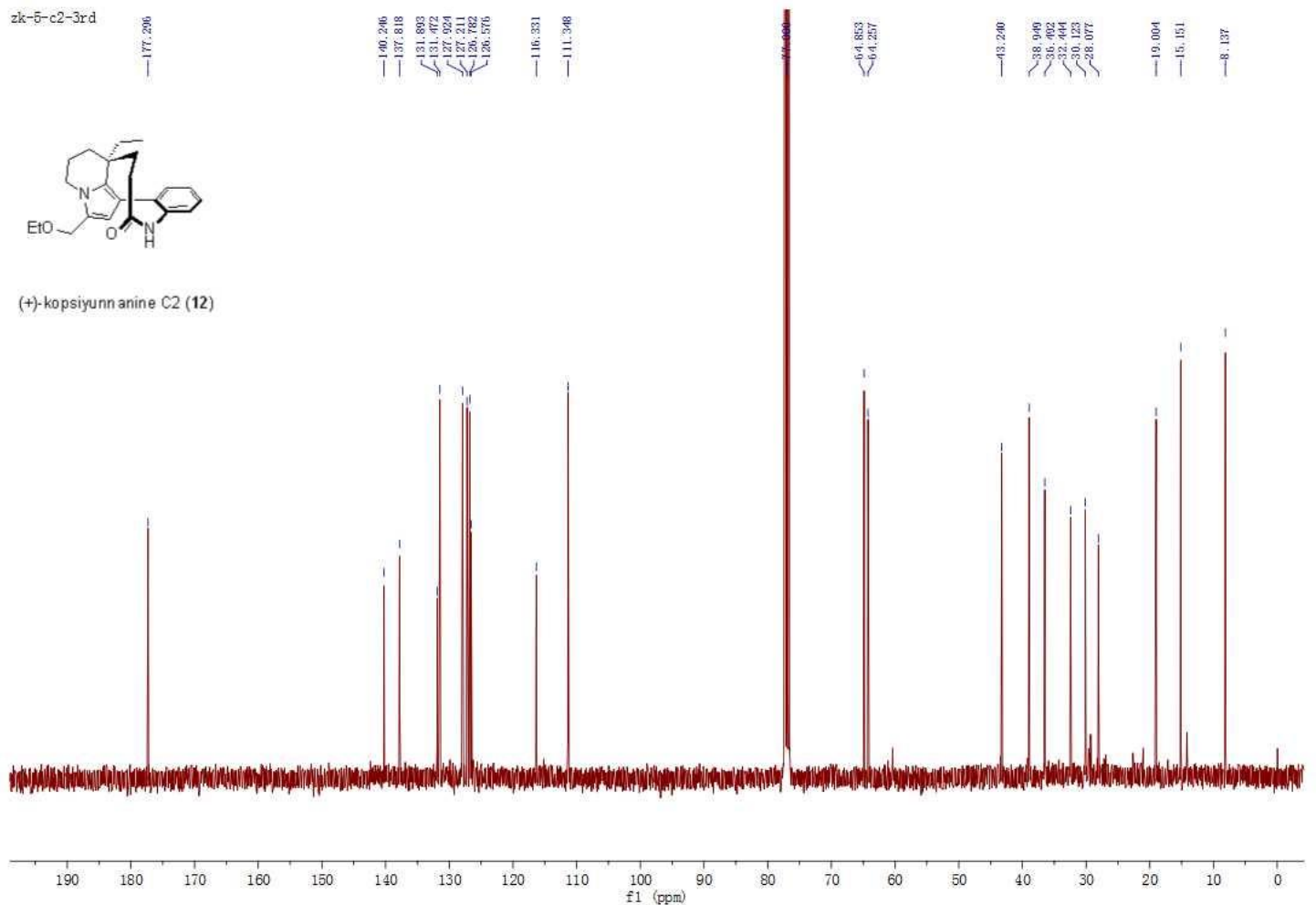
Data File F: \DATA $\backslash \mathrm{ZK} \backslash \mathrm{zk}-3-158-3(\mathrm{AD}, 85 \%, 1.0 \mathrm{ml}) . \mathrm{D}$

Sample Name: zk-3-158-3 (AD, 85\%,1.0ml)
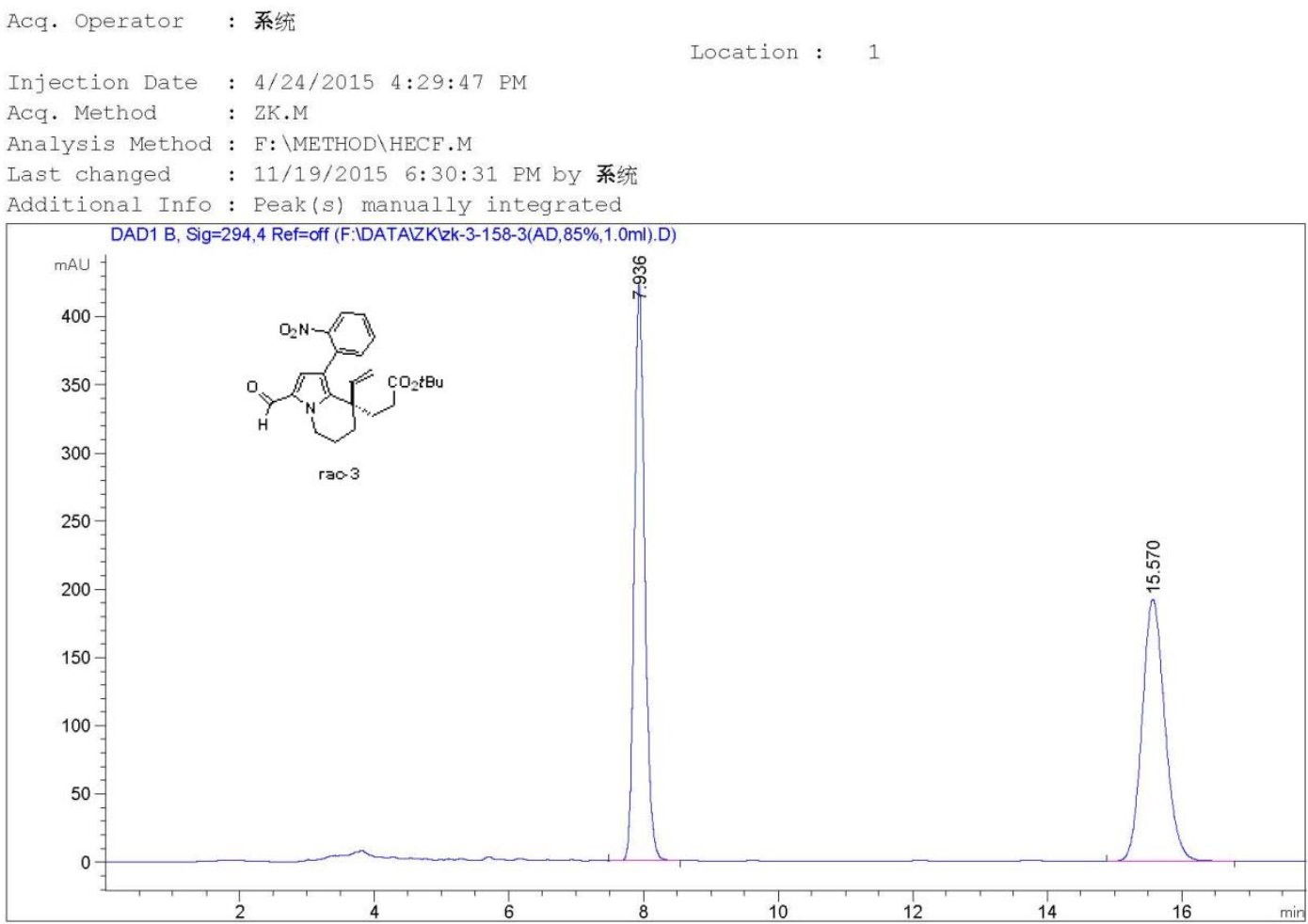

Area Percent Report

$\begin{array}{lll}\text { Sorted By } & : & \text { Signal } \\ \text { Multiplier } & : & 1.0000 \\ \text { Dilution } & : & 1.0000\end{array}$

Use Multiplier \& Dilution Factor with ISTDs

Signal 1: DAD1 B, Sig=294,4 Ref=off

\begin{tabular}{|c|c|c|c|c|c|c|}
\hline $\begin{array}{c}\text { Peak } \\
\quad \#\end{array}$ & $\begin{array}{l}\text { RetTime } \\
\text { [min] }\end{array}$ & Type & $\begin{array}{l}\text { Width } \\
{[\mathrm{min}]}\end{array}$ & $\begin{array}{c}\text { Area } \\
{\left[\mathrm{mAU}^{*} \mathrm{~S}\right]}\end{array}$ & $\begin{array}{l}\text { Height } \\
\text { [mAU] }\end{array}$ & $\begin{array}{c}\text { Area } \\
\frac{8}{8}\end{array}$ \\
\hline & & & & ----- & - & -- \\
\hline 1 & 7.936 & BB & 0.1650 & 4535.05518 & 423.14297 & 2818 \\
\hline
\end{tabular}

$\begin{array}{lllllll}2 & 15.570 & \mathrm{BB} & 0.3626 & 4484.22754 & 191.93056 & 49.7182\end{array}$

Totals : $\quad 9019.28271 \quad 615.07353$

$* * *$ End of Report *** 
Data File F: \DATA $\backslash \mathrm{ZK} \backslash \mathrm{xu}-1-65-1$ (AD, 85\%,1.0ml).D

Sample Name: $\mathrm{xu}-1-65-1(\mathrm{AD}, 85 \%, 1.0 \mathrm{ml})$
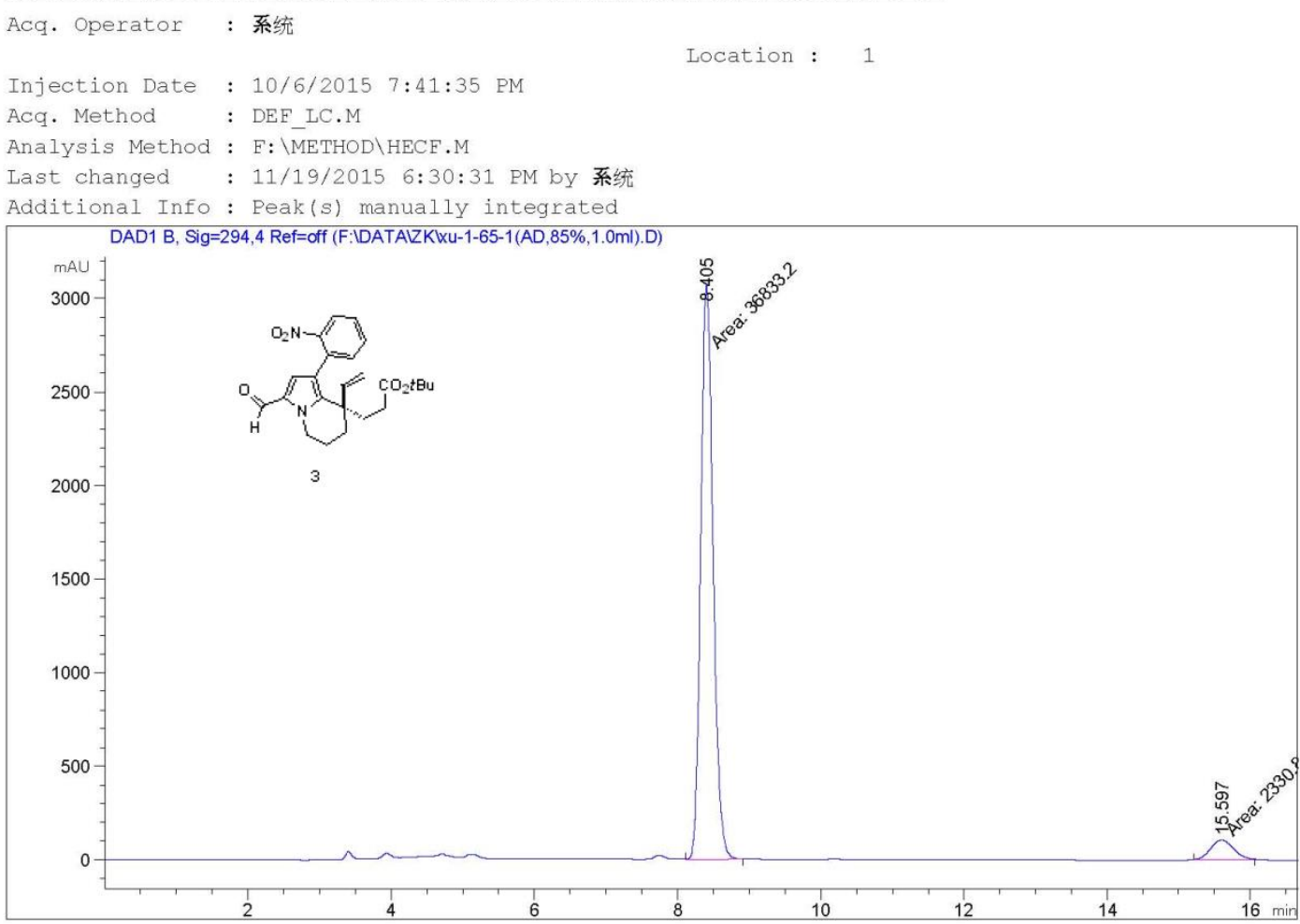

Area Percent Report

$\begin{array}{lll}\text { Sorted By } & : & \text { Signal } \\ \text { Multiplier } & : & 1.0000 \\ \text { Dilution } & : & 1.0000\end{array}$

Use Multiplier \& Dilution Factor with ISTDs

Signal 1: DAD1 B, Sig=294,4 Ref=off

\begin{tabular}{|c|c|c|c|c|c|c|}
\hline $\begin{array}{c}\text { Peak } \\
\text { \# }\end{array}$ & $\begin{array}{c}\text { RetTime } \\
\text { [min] }\end{array}$ & Type & $\begin{array}{l}\text { Width } \\
{[\mathrm{min}]}\end{array}$ & $\begin{array}{c}\text { Area } \\
{\left[\mathrm{mAU}{ }^{\star} \mathrm{s}\right]}\end{array}$ & $\begin{array}{l}\text { Height } \\
\text { [mAU] }\end{array}$ & $\begin{array}{c}\text { Area } \\
\frac{8}{8}\end{array}$ \\
\hline$\perp$ & 8.405 & MM & 0.2001 & $.68332 \mathrm{e} 4$ & 3067.81396 & 485 \\
\hline
\end{tabular}

$\begin{array}{llllll}2 & 15.597 \mathrm{MM} & 0.3706 & 2330.86353 & 104.82739 & 5.9515\end{array}$

Totals : $\quad 3.91640 \mathrm{e} 4 \quad 3172.64136$

$* * *$ End of Report *** 